\title{
Meeting abstracts of Colossal Facet Conference - 3rd World Congress on Cancer 2019 "New strategies to prevent, diagnose and treat Cancer based on Precision Medicine"
}

\author{
Prague, Czech Republic; 23-25 Sep 2019; Published: 27 Feb 2020 \\ Correspondence to: Prof. Ciro Isidoro, Department of Health Sciences, Università del Piemonte Orientale "A. Avogadro", Novara \\ 28100, Italy. E-mail: ciro.isidoro@med.uniupo.it
}

\section{Editorial Note}

This special issue of Journal of Cancer Metastasis and Treatment is dedicated to the proceedings of the 3rd World Congress on Cancer 2019 held in Prague (Czech Republic) during September 23-25 (http://colossalfacet.com/ cancer-conference/).

\begin{abstract}
The theme of the Conference Cancer 2019 was "New strategies to prevent, diagnose and treat Cancer based on Precision Medicine". The conference focused on the biomolecular mechanisms of cancer development, on the altered energetic metabolism in cancer cells, on the cancer patient's metabolic alterations, and on the diagnostic and therapeutic approaches. Major topics included: pathogenetic mechanisms (oncogenes, oncosuppressors, DNA repair, cancer stem cells, epigenetics, inflammation, and immune responses); metabolism (nutrition, fasting, and obesity) and cancer; novel strategies for prevention, diagnosis and therapy (imaging, phytochemicals, and nanotheranostics). The conference gathered academicians and young inspired scientists from all around the world with the aim to strengthen the international cooperation in the fight against cancer. One other major goal of this conference was, in fact, to create an atmosphere of interactions between young and senior scientists to favor novel cooperation that can bring new and more efficacious strategies to understand and fight cancer. The Organizing Scientific Committee was formed by renowned scientists in the field who delivered a keynote Speech. Thomas $\mathrm{N}$ Seyfried delivered the honorary lecture. In addition, we had invited lectures, oral communications, and poster presentation from registered attendees from all around the world.
\end{abstract}

This special issue collects the contribution in the form of original research papers or review articles from all participants at the conference.

Keywords: Autophagy, biomarkers, cachexia, cancer metabolism, cancer therapy, cell death, epigenetics, metastasis, tumor microenvironment, precision medicine 


\section{Amino acid depleting enzymes alone or in combinations as a therapeutic strategy for cancer treatment}

\section{John DiGiovanni}

Division of Pharmacology \& Toxicology, College of Pharmacy and Livestrong Cancer Institutes, Dell Medical School, University of Texas at Austin, Austin, TX 78712, USA.

Background and aim: Significant differences exist between the metabolism and antioxidant requirements of normal and malignant cells. Tumor cells depend on exogenous nutrients in their microenvironment to fulfill the elevated energy requirements and for maintaining appropriate intracellular antioxidant levels. Deprivation of amino acids results in growth inhibition or death of tumor cells by the modulation of various signaling cascades and in some cases redox balance. We have been evaluating potential therapeutic enzymes that degrade critical amino acids required for tumor growth. These engineered human enzymes include one that degrades either $L$-cysteine and one that degrades methionine.

Experimental procedures: (1) in vitro cell culture experiments to evaluate cell survival using 3-(4,5-dimethylthiazol-2yl)-2,5-diphenyltetrazolium bromide (MTT) and crystal violet assays; (2) metabolomics analyses of amino acids and metabolites; (3) analyses of oncogenic cell signaling, reactive oxygen species (ROS) levels, and DNA damage as well as cell cycle changes using flow cytometry; (4) in vivo allograft and xenograft tumor experiments with various cancer cell lines.

Results: Depletion of extracellular $L$-cys/cystine led to depletion of intracellular $L$-cys, decreased levels of intracellular glutathione (GSH), and increases in intracellular ROS leading to activation of cellular signaling pathways, oxidative DNA damage, and ultimately cancer cell death. Cyst(e)inase, given i.p., significantly reduced serum levels of $L$-cys and significantly inhibited tumor growth in vivo of both prostate and pancreatic cancer xenograft and allograft tumor models. Notably, targeting a second antioxidant pathway together with cyst(e)inase (i.e., the thioredoxin pathway) using a thioredoxin reductase inhibitor led to synergistic cancer cell killing and also sensitized tumor cells found to be more resistant to cyst(e) inase alone. These and other studies on the mechanisms associated with the potential anticancer activity of Cyst(e)inase are presented. In addition, we have also studied the potential therapeutic application of a human engineered methionine ( $L$-met) degrading enzyme called methionine gamma lyase (hMGL). Both mechanistic studies as well as in vivo preclinical therapeutic studies demonstrate significant efficacy against several cancers with hMGL.

Conclusion: Depletion of amino acids such as $L$-cys and $L$-met using human engineered enzymes offer novel approaches for treating cancer either given alone or more likely in combination with other agents.

\section{Investigating metabolic cancer vulnerabilities by high-content metabolomic screening}

\section{Stefano Tiziani}

Department of Nutritional Sciences, University of Texas at Austin, Austin, TX 78712, USA.

Background and aim: The important role of cell metabolism in furthering cancer development and growth is increasingly recognized ${ }^{[1,2]}$. Recent advances in high-throughput metabolomics technology are leading to 
its growing role during the development of new drugs targeting metabolic vulnerabilities in multiple cancer diseases $^{[3,4]}$. However, drug discovery is limited by the unsuitability of animal models for high-throughput drug screening. Moreover, animal studies may not adequately predict the clinical efficacy of therapeutics in humans. These limitations motivated researchers to develop new three-dimensional (3D) in vitro models to better mimic the in vivo tumor microenvironment ${ }^{[5]}$.

Experimental procedure: Here, we introduce a novel high-content metabolomics screen based on highresolution direct infusion mass spectrometry (DIMS) technology able to monitor the metabolic response of drug-treated mammalian cells in 3D 96-well format. This rapid and systematic metabolomic method was validated on multiple cancer and normal cells, cultured either in individual or in co-culture cell systems using $13 \mathrm{C}-15 \mathrm{~N}$ labeled tracer analysis.

Results: Novel synergistic combination of drugs were identified utilizing the metabolic profiling obtained using DIMS. These include chemotherapies targeting the metabolic reprogramming of cancer cells, including mitochondrial oxidative phosphorylation and glutaminolysis.

Conclusion: Overall, the rapid data acquisition and improved detection limits of mass spectrometry are paving the way for applications of metabolomics in preclinical screening ${ }^{[5,6]}$, opening new opportunities in drug discovery and personalized medicine.

\title{
REFERENCES
}

1. Luengo A, Gui DY, Vander Heiden MG. Targeting metabolism for cancer therapy. Cell Chem Biol 2017; 24:1161-80.

2. Molina JR, Sun Y, Protopopova M, Gera S, Bandi M, et al. An inhibitor of oxidative phosphorylation exploits cancer vulnerability. Nat Med 2018;24:1036-46.

3. Tiziani S, Kang Y, Choi JS, Roberts W, Paternostro G. Metabolomic high-content nuclear magnetic resonance-based drug screening of a kinase inhibitor library. Nat Commun 2011;2:545.

4. Wishart DS. Emerging applications of metabolomics in drug discovery and precision medicine. Nat Rev Drug Discov 2016;15:473-84.

5. Lu X, Lodi A, Konopleva M, Tiziani S. Three-dimensional leukemia co-culture system for in vitro high-content metabolomics screening. SLAS Discov 2019;24:817-28.

6. Lodi A, Saha A, Lu X, Wang B, Sentandreu E, et al. Combinatorial treatment with natural compounds in prostate cancer inhibits prostate tumor growth and leads to key modulations of cancer cell metabolism. NPJ Precis Oncol 2017;1:pii:18.

\section{Clinical application of artificial intelligence in ovarian cancer}

\author{
Se Ik Kim¹, Youngjin Han², Untack Cho', Yong Sang Song ${ }^{1,2}$ \\ ${ }^{1}$ Department of Obstetrics and Gynecology and Cancer Research Institute, College of Medicine, Seoul National \\ University, Seoul 03080, Korea. \\ ${ }^{2}$ Biomodulation, Department of Agricultural Biotechnology, Seoul National University, Seoul 08826, Korea.
}

Among several types of gynecologic cancer, ovarian cancer is the most lethal type. Due to the absence of specific symptoms and effective biomarkers, the survival rate of ovarian cancer is poor. Moreover, platinum resistance is a major obstacle in ovarian cancer treatment. Thus, accurate biomarkers associated with chemoresistance and recurrence of the cancer are necessitated. To establish personalized therapeutic strategies for ovarian cancer patients, a prediction model that precisely predicts patient responses to chemotherapy and diagnosis could be computed and incorporated. Although many prediction models for cancer have been suggested, few models specific for ovarian cancer have been proposed. Thus, we performed integrative analysis incorporating both clinico-pathologic and multi-omics data and developed prediction models for diagnosis and prognosis of ovarian cancer. In addition, we conducted metagenome 
analysis of patients with benign tumors and ovarian cancer to construct an early detection model of ovarian cancer. Gene expression data from TCGA database have been analyzed using the deep neural network model to develop a model for predicting platinum-sensitivity in high-grade serous ovarian cancer. Lastly, we integrated clinico-pathologic and multi-omics data to reveal multiple factors associated with ovarian cancer progression.

\title{
4. Genetic and epigenetic regulation of therapy resistance in ovarian cancer by long non- coding RNAs
}

\author{
Danny N. Dhanasekaran ${ }^{1}$, Ji Hee Ha ${ }^{1}$, Ranagasudhagar Radhakrishanan ${ }^{1}$, Muralidharan \\ Jayaraman ${ }^{1}$, Ciro Isidoro ${ }^{2}$, Yong Sang Song ${ }^{3}$ \\ ${ }^{1}$ Stephenson Cancer Center, University of Oklahoma Health Sciences Center, Oklahoma City, OK 73104, USA. \\ ${ }^{2}$ Università del Piemonte Orientale, Novara 28100, Italy. \\ ${ }^{3}$ Seoul National University, Seoul 03080, Korea.
}

Background and aim: Ovarian cancer remains the most fatal gynecological cancer in the world, with a fiveyear survival rate of only $46 \%$ for the localized disease and $29 \%$ for the distant-stage disease. With the recent analysis of cancer genome, long non-coding RNAs (lncRNAs) are emerging as critical players in the pathobiology of many cancers, thus identifying them as new genomic targets for precision cancer medicine. Therefore, we sought to identify the critical $\operatorname{lncRNA}(\mathrm{s})$ involved in ovarian cancer genesis, progression, therapy resistance, and disease recurrence.

Experimental procedure: To identify the lncRNAs critically involved in ovarian cancers, we carried out a global analysis of mRNAs as well as lncRNAs that are differentially expressed in patient-derived ovarian cancer cells, using a series of biased and unbiased transcriptome analyses.

Results: The results indicate 1351 lncRNAs and 1591 mRNAs were significantly dysregulated in patientderived cancer cells compared to normal fallopian tube-derived epithelial control cells. Co-expression network analysis of coding and noncoding RNAs identified the etiological role for several, thus far, unidentified lncRNAs and mRNAs in ovarian cancer. Further analyses indicated that the lncRNA-regulated gene expression network in ovarian cancer involves both genetic and epigenetic mechanisms.

Conclusion: Our findings with representative lncRNAs indicate that they can serve as a novel diagnostic as well as prognostic biomarkers. In addition, the findings that the silencing of specific lncRNAs inhibits xenograft tumor growth identify them as new therapeutic targets in ovarian cancer.

\section{Radiotherapy and immunotherapy promote tumoral lipid oxidation and ferroptosis}

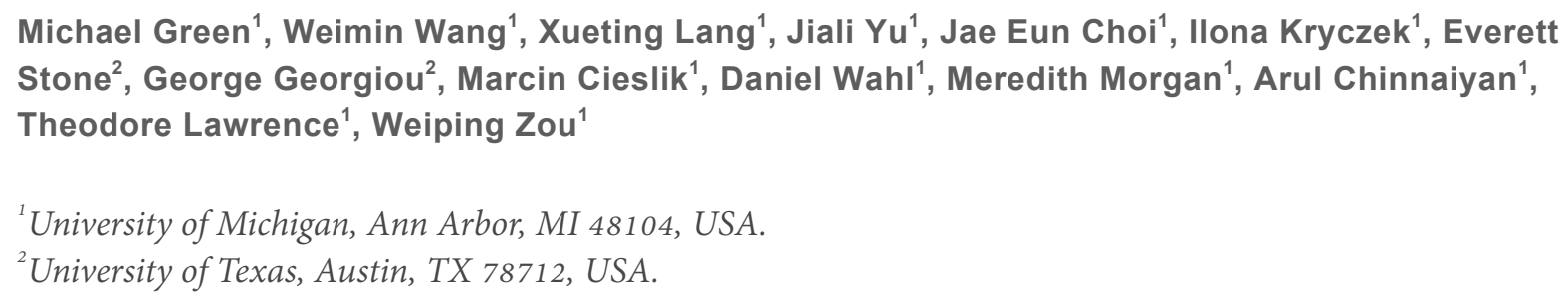

Background and aim: Cancer immunotherapy restores or enhances the effector function of CD8+ T cells in the tumor microenvironment. Radiotherapy can indirectly stimulate CD8+ T cell function through innate immune signaling. Direct connections between radiotherapy and adaptive CD8+ $\mathrm{T}$ cell function 
remain undefined. Ferroptosis is a recently discovered form of cell death and results from iron-dependent accumulation of lipid peroxides. It is unclear whether, and how, ferroptosis is involved in T cell immunity, cancer immunotherapy, and radiotherapy efficacy.

Experimental procedure: To understand the importance of ferroptosis in immunotherapy and radiotherapy efficacy, we used genetic deletion of key ferroptosis effector genes and pharmacologic agonists and antagonists. Lipid oxidation was quantified using C11-BODIPY. A wide variety of in vivo and ex vivo analyses was performed in tumor and immune cells.

Results: We show that immunotherapy-activated CD8+ T cells and radiotherapy enhance ferroptosisspecific lipid peroxidation in tumor cells, and that increased ferroptosis contributes to the anti-tumor efficacy of immunotherapy and radiotherapy. Mechanistically, interferon gamma (IFN $\gamma$ ) released from CD8+ T cells downregulates the expression of SLC7A11, a subunit of the glutamate-cystine antiporter system xc-, impairs the uptake of cystine by tumor cells, and, as a consequence, promotes tumor cell lipid peroxidation and ferroptosis.

Conclusion: This work establishes a novel mechanism through which CD8+ T cells function. This work expands our understanding of the interactions between immunotherapy and radiotherapy.

\section{Polyploidy and the origin of human tumors}

\section{Jinsong Liu}

The University of Texas, MD Anderson Cancer Center, Houston, TX 77030, USA.

Polyploid giant cancer cells (PGCCs) have long been observed in cancer and were thought originally to be nondividing. Surprisingly, the formation of blastomere by cleavage division after the formation of the zygote, with progressive decrease in cell size and increase in nuclear to cytoplasmic ratio, is the first step in embryogenesis, also shows abundant polyploidy. The evidence from our laboratories demonstrated that the stress-induced PGCCs can divide by endoreplication (endocycle and endomitosis), which leads to increased nuclear to cytoplasmic ratio, in turn leading to dedifferentiation of somatic cells and acquisition of embryonic stemness. Therefore, formation of PGCCs in somatic cells may represent a previously overlooked endogenous embryonic program that can be activated to dedifferentiate somatic cells into stem cells of various potencies for tumor initiation. Based on these data, I propose that human tumors originate from stem cells at a specific developmental hierarchy, which can be achieved by dualistic origin: dedifferentiation of the zygote (sexual) via the blastomeric-mediated cleavage division during normal development or transformation from damaged or aged mature somatic cells via a blastomericlike embryonic program (asexual) via formation of PGCCs. Initiation of the tumor begins with stem cells that have uncoupled the differentiation from the proliferation program, which results in stem cell maturation arrest. Thus, the birth of a tumor can be viewed as a triad that originates from stem cells via dedifferentiation through a blastomeric or blastomeric-like program, differentiation along Waddington's landscape, and arrested at a specific developmental hierarchy. The significance of polyploid blastomere-like cancer stem cells in cancer therapy is discussed.

\section{Ketogenic diet as a cancer treatment: in vitro quantification}

\section{Edward Henry Mathews, George Edward Mathews, Albertus Abram Meyer}


Background: The glucose deprivation Restricted Ketogenic diet (KD-R) in combination with Metformin use, is a non-toxic broad-spectrum approach that targets the important metabolic differences between normal and cancer cells. The optimal use of this approach for cancer treatment is investigated using in vitro tests.

Method: Tests were carried out at 3-mmol/L blood glucose (BG) to mimic the BG effect of KD-R in combination with Metformin. Two breast and one cervical cancer as well as one non-tumorigenic cell were used.

Results: The different cell lines were affected differently. This suggests that glucose deprivation via KD-R and Metformin will not equally affect different cancers. All cell lines were most adversely affected after three weeks. Cell growth decreased to $32 \%$ for the most glucose avid cancer cell line.

Partial recovery occurred after three months. Full cancer extinction can thus not be reached with only KD-R and Metformin. Adjuvant treatments are needed. These treatments should be done when the cancer cells are at their most vulnerable, i.e., three weeks after reaching a BG level of $3 \mathrm{mmol} / \mathrm{L}$.

Future work: Future work will entail adjuvant treatments such as chemotherapy together with KD-R and Metformin. Results should be available before the conference. The focus of our conference presentation is on the latest results.

\section{DNA repair and damage response in personalized brain cancer chemotherapy}

\section{Bernd Kaina, Yang He, Oliver Switzeny, Wynand P. Roos, Markus Christmann, Thomas Hofmann}

Institute of Toxicology, University Medical Center Mainz, Mainz D-55130, Germany.

Background and aim: The first-line chemotherapeutic for malignant glioma is the DNA methylating agent temozolomide. The mechanism of cell death triggered by the minor DNA lesion, $\mathrm{O}^{6}$-methylguanine, induced by the agent is well described. It rests on conversion of the lesion through mismatch repair into DNA double-strand breaks (DSB) that trigger downstream pathways including apoptosis and senescence ${ }^{[1]}$. Consequently, corresponding repair pathways are expected to have a great impact on temozolomide resistance, and evidence was provided for the involvement of $\mathrm{O}^{6}$-methylguanine DNA methyltransferase (MGMT), mismatch repair, and DSB repair by homologous recombination through BRCA2 and Rad $51^{[2]}$ as well as XRCC $3^{[3]}$. However, only MGMT found the way into the clinic, being used as predictor for therapy outcome $e^{[4]}$.

Experimental procedure: A battery of cell und molecular biological methods was applied, including apoptosis, senescence, and autophagy measurements.

Results: We compared methods of determining the MGMT promoter methylation status, which corresponds to MGMT silencing and therapy, and showed that MS-HRM is superior compared to methylation-specific polymerase chain reaction ${ }^{[5]}$. We also show that downstream of $\mathrm{O}^{6}$-methylguanine derived DSBs are ATR/ ATM triggered pathways that activate apoptosis and senescence. Thus, data are shown demonstrating that the SIAH1-HIPK2-p53ser46 pathway plays a key role in regulating temozolomide-induced apoptosis ${ }^{[6]}$. The question of temozolomide threshold doses in activating survival and death pathways is also addressed ${ }^{[7]}$.

Conclusion: MGMT, mismatch repair and the SIAH1-HIPK2-p53ser46 pathway are key elements in personalized glioblastoma therapy with DNA-alkylating drugs. 


\section{REFERENCES}

1. Knizhnik AV, Roos WP, Nikolova T, Quiros S, Tomaszowski KH, et al. Survival and death strategies in glioma cells: autophagy, senescence and apoptosis triggered by a single type of temozolomide-induced DNA damage. PLoS One 2013;8:e55665.

2. Quiros S, Roos WP, Kaina B. Rad51 and BRCA2--New molecular targets for sensitizing glioma cells to alkylating anticancer drugs. PLoS One. 2011;6:e27183.

3. Roos WP, Frohnapfel L, Quiros S, Ringel F, Kaina B. XRCC3 contributes to temozolomide resistance of glioblastoma cells by promoting DNA double-strand break repair. Cancer Lett 2018;424:119-26.

4. Wiewrodt D, Nagel G, Dreimüller N, Hundsberger T, Perneczky A, et al. MGMT in primary and recurrent human glioblastomas after radiation and chemotherapy and comparison with 553 status and clinical outcome. Int J Cancer 2008;122:1391-9.

5. Switzeny OJ, Christmann M, Renovanz M, Giese A, Sommer C, et al. MGMT promoter methylation determined by HRM in comparison to MSP and pyrosequencing for predicting high-grade glioma response. Clin Epigenetics 2016;8:49.

6. He Y, Roos WP, Wu Q, Hofmann TG, Kaina B. The SIAH1-HIPK2-p53ser46 damage response pathway is involved in temozolomideinduced glioblastoma cell death. Mol Cancer Res 2019;17:1129-41.

7. He Y, Kaina B. Are There Thresholds in glioblastoma cell death responses triggered by temozolomide? Int J Mol Sci 2019;20:pii: E1562.

\section{Metabolic management of glioblastoma}

\section{Thomas Nicholas Seyfried}

Department of Biology, Boston College, Chestnut Hill, MA 02467, USA.

Glioblastoma multiforme (GBM) remains among the most aggressive and difficult to manage primary brain tumors in humans. Abnormalities in the number, structure, and function of GBM mitochondria compromise energy metabolism through OxPhos. Glucose and glutamine are recognized as the major fermentable fuels that drive GBM growth through glycolysis and glutaminolysis, respectively. The glutamine antagonist, 6-diazo-5-oxo- $L$-norleucine (DON), was administered together with a calorically restricted ketogenic diet (KD-R) to treat late-stage orthotopic growth in two syngeneic mouse models of GBM: the highly invasive mesenchymal tumor, VM-M3, and the high-grade stem cell glioma, CT-2A. DON targets glutaminolysis while KD-R reduces glucose and, at the same time, elevates neuroprotective and nonfermentable ketone bodies. The diet/drug therapeutic strategy caused massive tumor cell death or mitotic arrest, while reversing disease symptoms and improving overall survival without toxicity. The therapeutic strategy also reduced edema, hemorrhage, and inflammation associated with rapid tumor growth. Moreover, the KD-R diet facilitated DON delivery to the brain and allowed a lower nontoxic dosage to achieve therapeutic effect. Data from human case reports are also presented. These findings support the importance of glucose and glutamine in driving GBM growth and provide a plausible therapeutic strategy for the non-toxic metabolic management of GBM and any cancer with mitochondrial defects.

\section{Novel perspectives on the immune environment of acute myeloid leukemia using multiomyx}

Josette William, Nicholas Hoe, Mate Nagy, Raghav Padmanabhan, Qingyan Au

NeoGenomics, Aliso Viejo, CA 92656, USA.

Background and aim: Acute myeloid leukemia (AML) is a clinically and molecularly heterogeneous disorder. Despite its poor prognosis, the treatment of AML remains largely unchanged over the past several decades with high-dose chemotherapy remaining the mainstay of therapy. This has led to interest in exploring novel therapeutic approaches, such as bispecific antibodies, chimeric antigen receptor $\mathrm{T}$ cells, tumor vaccines, and immune checkpoint inhibitors. 
The bone marrow (BM) constitutes the home niche for leukemic cells. Tumor microenvironment (TME) is defined as the cellular environment in which the tumor exists. This environment is made up of endothelial, stromal, and immune cells and plays a key role in the development, propagation, and survival of cancer cells. The immune microenvironment has been well described in several hematologic malignancies, including Hodgkin lymphoma, acute lymphoblastic leukemia, chronic myeloid leukemia, and chronic lymphocytic leukemia, but less is known about the microenvironment in AML.

We studied the myeloid subsets in bone marrow tissues of normal and AML patients using MultiOmyx technique. We aimed to clarify the clinical significance of these cells in the AML patients.

Experimental procedure: MultiOmyx is an exclusive proprietary multiplex immunofluorescent technology that overcome the challenges for immuno-oncology biomarker profiling. It enables detection and visualization of up to 60 biomarkers on a single formalin-fixed paraffin-embedded (FFPE) slide and co-expression analysis of up to 25 stains on a single cell, which is unattainable with the conventional immunohistochemistry (IHC) technique. Other advantages include: quantitative single cell classification, measures of marker intensity (mean, median, and total), and full spatial context for measuring the distances between cells with different immunophenotypes.

Results: Myeloid subsets present in tumors are heterogeneous and play a crucial role in promoting cancer development and metastasis. Tumor associated macrophages (TAMs) and myeloid-derived suppressor cells (MDSCs) all contribute to an immunologically permissive microenvironment for cancer cells. Based on surface markers expression, MDSC can be further subdivided into granulocytic MDSC (G-MDSC, polymorphonuclear MDSC) and monocytic MDSC (M-MDSC). MDSC have also been shown to express immune checkpoint ligands such as programmed death-ligand 1 (PD-L1) that can suppress $\mathrm{T}$ cell responses in vitro. There is little information regarding MDSCs in AML. TAMs can be polarized by signals from their environment into two major subsets, called M1 and M2 macrophages. Acute myeloid leukemia blasts have been shown to differentiate monocytes from healthy donors into an M2-like phenotype in transwell coculture assays. In our study, we were able to highlight the immune landscape of AML and compared it with the landscape for normal bone marrow. We observed that both M-MDSC and G-MDSC accumulated within the TME in AML BM samples, with higher frequency of G-MDSCs over M-MDSCs. The data also reveal abundant M2 macrophages present in the TME of the AML samples. The detection of both MDSCs and M2 macrophages in these samples supports the hypothesis that these cells contribute to the establishment of an immunosuppressive TME. Using the MultiOmyx proprietary algorithm, which takes into account the staining patterns, we quantified the counts and density of different tumor-resident myeloid subsets and measured the spatial distance from the different subsets of tumor-resident myeloid cells to CD34+ blasts in AML samples. We highlighted the correlations between the immunosuppressive myeloid cells and the different subsets of T cells including T-regulatory cells in AML clinical biopsy samples. TAMs and MDSCs are emerging as potential biomarkers for diagnosis and prognosis of cancer as well as therapeutic targets of many immunomodulating agents. As demonstrated in this study, MultiOmyx multiplexed panel has the potential to monitor the changes of immunosuppressive myeloid cells in response to immune modulating drugs such as MDSC-targeting drugs (e.g., PDE-5 inhibitors and COX-2 inhibitors), TAM-targeting agents (e.g., anti-CSF1R), and combination therapy in treatment of AML.

\section{Radiation oncology updates in treatment of prostate cancer}

\section{Lauren Layer Mayo}

The University of Texas MD Anderson Cancer Center, Houston, TX 77030, USA. 
I am a radiation oncologist at MD Anderson Cancer Center with a large proportion of my practice dedicated to prostate cancer. I would like to update the audience with the basic algorithms of radiation, hormonal, and systemic therapy for low, intermediate, and high risk. This talk would include information on active surveillance, definitive radiation treatment, brachytherapy as single modality and boost treatment, adjuvant and salvage (post-operative) radiation therapy, and hypofractionation and stereotactic body radiation therapy. I understand the audience is a variety of clinicians in the medical field. I would tailor the talk to provide an overview so all can understand what is available to all prostate cancer patients and the updated studies to support these recommendations.

\title{
12. Novel DNA modification in cancer
}

Tao P. Wu ${ }^{2,6, \#}$, Qi Xie ${ }^{1, \#}$, Ryan C. Gimple ${ }^{1,3, \#}$, Zheng $\mathrm{Li}^{2}$, Qiulian $\mathrm{Wu}^{1}$, Yang $\mathrm{Yu}^{4}$, Yinsheng Wang ${ }^{4}$, Shideng Bao ${ }^{5}$, Andrew Z. Xiao' ${ }^{2}$, Jeremy N. Rich ${ }^{1}$

\author{
${ }^{1}$ Department of Medicine, Division of Regenerative Medicine, University of California, San Diego, La Jolla, CA \\ 92037, USA. \\ ${ }^{2}$ Department of Genetics and Yale Stem Cell Center, Yale School of Medicine, New Haven, CT 06520, USA. \\ ${ }^{3}$ Department of Pathology, Case Western Reserve University, Cleveland, OH 44120, USA. \\ ${ }^{4}$ Department of Chemistry, University of California, Riverside, Riverside, CA 92521, USA. \\ ${ }^{5}$ Department of Stem Cell Biology and Regenerative Medicine, Lerner Research Institute, Cleveland Clinic, \\ Cleveland, OH 44195, USA. \\ ${ }^{6}$ Present address: Department of Molecular and Human Genetics, Baylor College of Medicine, One Baylor \\ Plaza, Houston, TX 77030, USA. \\ ${ }^{\#}$ Co-equal first authors.
}

Genetic drivers of cancer can be dysregulated through epigenetic modifications of DNA. Although the critical role of DNA 5-methylcytosine $(5 \mathrm{mC})$ in the regulation of transcription is recognized, the functions of other non-canonical DNA modifications remain obscure.

We report the identification of novel DNA N6-methyladenine (N6-mA) modifications in human tissues and implicate this epigenetic mark in human disease, specifically the highly malignant brain cancer glioblastoma. Glioblastoma markedly upregulated N6-mA levels, which co-localized with heterochromatic histone modifications, predominantly H3K9me3. N6-mA levels were dynamically regulated by the DNA demethylase ALKBH1, depletion of which led to transcriptional silencing of oncogenic pathways through decreasing chromatin accessibility. Targeting the N6-mA regulator ALKBH1 in patient-derived human glioblastoma models inhibited tumor cell proliferation and extended the survival of tumor-bearing mice, supporting this novel DNA modification as a potential therapeutic target for glioblastoma.

Furthermore, ALKBH1 controls the hypoxia responding genes in glioblastoma. Collectively, our results uncover a novel epigenetic node in cancer through the DNA modification N6-mA. The regulators of this new modification could serve as novel therapeutic targets in cancer therapy.

\section{Evaluation of undiagnosed liver masses that do not exhibit typical imaging features, including even Stage C HCC}

\section{Metin Basaranoglu}

Bezmialem Vakif University, İstanbul 34093, Turkey. 
Background and aim: Sometimes, despite all blood examinations, transabdominal ultrasound, and computerized tomography with or without magnetic resonance imaging by multidisciplinary approach, we cannot reach a strict diagnosis in patients with liver masses. In this study, we aimed to evaluate undiagnosed liver masses that do not exhibit typical imaging features.

Material and methods: In this study, we retrospectively evaluated 140 patients with undiagnosed liver mass(es) without any typical imaging features. Then, percutaneous liver biopsy by transabdominal ultrasound guiding was performed in 121 patients to obtain a liver specimen. A single gastroenterologist who has much experience with radiologic biopsies performed all biopsies in this study. This study included the years 2011-2013. A single experienced radiologist reevaluated images from the records in 2014.

Results: A pathologist evaluated 121 patients' liver specimens. The distribution of the diagnosis is as follows: 45 patients with metastasis, 24 patients with hepatocellular carcinoma (HCC), 16 patients with nothing, 8 patients with advanced stage chronic liver disease, 5 patients with neuroendocrine tumours (NET), 5 patients with dysplastic nodule or well-differentiated HCC, 4 patients with cholangiocarcinoma, 4 patients with pseudotumor (secondary to infections), 2 patients with steatosis, 2 patients with hemangioma, 1 patient with steatohepatitis, 1 patient with extramedullar hematopoiesis, 1 patient with necrotizing granuloma, 1 patient with biliary cirrhosis (sistozomiazis), 1 patient with cyst hydatid, and 1 patient with mixed tumor (HCC + cholangiocarcinoma). The radiologist reevaluated the radiologic records of 70 patients. The distribution of these patients is as follows: 27 patients with HCC, 11 patients with chronic liver disease findings without any mass, 12 patients with metastasis, 6 patients with cholangiocarcinoma, 3 patients with hemangioma, 5 patients with abscess ( 1 with fasciola and 1 with cyst hydatid), 2 patients without any liver abnormality, 1 patient with dysplastic nodule, 1 patient with angiomyolipoma, 1 patient with gallbladder tumor, and 1 patient with focal nodular hyperplasia. A further distribution of the 27 patients with HCC is shown in Table 1 according to the Barcelona Clinic Liver Cancer Staging System.

Discussion: Our results show that HCC, even with Stage C, is one of the major causes of the liver masses that do not exhibit typical imaging features. $\mathrm{HCC}$ due to none $\mathrm{B}$ and none $\mathrm{C}$ was also a significant portion in this group of patients. More than half of the patients with HCC had normal serum $\alpha$-fetoprotein level even in HCC patients with Stage C. As expected, life expectancy was related to the stage of the disease.

\section{Therapeutic effects of trehalose liposomes against tumors along with apoptosis}

\section{Yoko Matsumoto}

Sojo University, Department of Life Sciences, Kumamoto 860-0082, Japan.

Background and aim: Trehalose stabilizes membranes and proteins in cells most likely by hydrogen bonding. In this study, inhibitory effects of trehalose liposomes (DMTre) composed of $L$ - $\alpha$ dimyristoylphosphatidylcholine (DMPC) and trehalose micelles (TreC14) on the growth of tumors along with apoptosis were obtained in vitro and in vivo.

Experimental procedure: DMTre were prepared by the method of sonication of a mixture of DMPC and TreC14 in a buffer solution with no organic solvent ${ }^{[1,2]}$. The thickness of fixed aqueous layer (TFAL) of DMTre was evaluated from the zeta potential by an electrophoretic light scattering measurement. The fusion and accumulation of DMTre in tumor cell membrane including a fluorescence probe was observed using confocal laser microscopy. Activation of caspases for tumor cells induced by DMTre was analyzed using a flow cytometer. Assessment of therapeutic effects of DMTre against xenograft mice and orthotropic graft bearing mice model of carcinoma was performed. 
Results: Hydrodynamic diameter $\left(d_{\text {hy }}\right)$ of DMTre composed of 30 mol\% DMPC and 70 mol\% TreC14 was $100 \mathrm{~nm}$ with single and narrow range of size distribution, which can avoid reticular endothelial system in vivo. An increase in TFAL values of DMTre was obtained in a dose-dependent manner. DMTre inhibited the growth of breast and lung tumor cells leading to apoptosis with the activation of caspases. The suppression of tumor weight of xenograft mice model of carcinoma treated with DMTre after inoculation with breast tumor cells was obtained along with apoptosis. A remarkable reduction of volume and weight in subcutaneous tumors on subcutaneous lung carcinoma-bearing mice administered with DMTre was obtained.

Conclusion: Anti-tumor activities of DMTre against carcinoma-bearing mice along with apoptosis were obtained. The results of this study could contribute to the development of therapeutic agents for patients with carcinoma in future clinical application.

\section{REFERENCES}

1. Matsumoto Y, Cao E, Ueoka R. Growth inhibition by novel liposomes including trehalose surfactant against hepatocarcinoma cells along with apoptosis. Anticancer Res 2013;33:4727-40.

2. Matsumoto Y, Kuwabara K, Ichihara H, Kuwano M. Therapeutic effects of trehalose liposomes against lymphoblastic leukemia leading to apoptosis in vitro and in vivo. Bioorg Med Chem Lett 2016;26:301-5.

\section{The effect of interactions between temozolomide and dexamethasone on the profile of 84 selected proteins in glioblastoma multiforme cells}

\section{Anna Bielecka-Wajdman, Tomasz Ludyga, Ewa Obuchowicz}

Department of Pharmacology, School of Medicine in Katowice, Medical University of Silesia, Katowice 40-055, Poland.

Background and aim: In patients with glioblastoma multiforme (GBM), standard chemotherapy with temozolomide (TMZ) is always supplemented with dexamethasone (DXM). Even though for years DXM has been applied as a "gold standard" in therapy of vasogenic edema, intracranial pressure, and mass effect, recent controversial results have challenged the widely accepted dogma concerning its using in therapy of GBM. The results of experimental studies emphasize that DXM may increase the aggressiveness of GBM by promoting the proliferation and invasiveness of cancer cells.

The aim of our study conducted on two primary glioblastoma lines obtained from patients and on the commercial line T98G was to assess the effects of TMZ and DXM, as well as their interaction, on the profile of 84 proteins involved in the process of carcinogenesis. The tests were performed using the Proteome Profiler Human XL Oncology Array Kit (R \& D) in cells cultured under two oxygen conditions: physiological for tumor hypoxia (2.5\% oxygen) or in standard laboratory conditions (20\% oxygen) frequently used in in vitro studies.

Results: Our results confirm the pro-tumorigenic properties of DXM but they also show that the response of GBM commercial and primary cell lines to DXM given to culture medium with or without TMZ is variable and depends on oxidation of the microenvironment.

Conclusion: It can be concluded that DXM and TMZ administered together or separately may induce different effects which depend on the degree of hypoxia prevailing in the malignant brain tumor. 


\section{Sensitive detection of metabolic abnormalities in adult T-cell leukemia/lymphoma and} induction of specific leukemic cell death using photodynamic therapy

Takashi Oka ${ }^{1,2}$, Hajime Mizuno ${ }^{3,7}$, Masumi Sakata ${ }^{3}$, Hirofumi Fujita ${ }^{4}$, Tadashi Yoshino ${ }^{1}$, Yoshihisa Yamano $^{5}$, Kozo Utsumi ${ }^{4}$, Tsutomu Masujima ${ }^{3}$, Atae Utsunomiya ${ }^{6}$

${ }^{1}$ Department of Pathology and Oncology, Graduate School of Medicine, Dentistry and Pharmaceutical Sciences, Okayama University, Okayama 700-8558, Japan.

${ }^{2}$ Department of Hematology, Oncology and Respiratory Medicine, Graduate School of Medicine, Dentistry and Pharmaceutical Sciences, Okayama University, Okayama 700-8558, Japan.

${ }^{3}$ Laboratory for Single Cell Mass Spectrometry, RIKEN Quantitative Biology Center (QBiC), Osaka 565-0874, Japan.

${ }^{4}$ Department of Cytology and Histology, Graduate School of Medicine, Dentistry and Pharmaceutical Sciences, Okayama University, Okayama 700-8558, Japan.

${ }^{5}$ Department of Rare Diseases Research, Institute of Medical Science, St. Marianna University School of Medicine, Kawasaki 216-8512, Japan.

${ }^{6}$ Department of Hematology, Imamura General Hospital, Kagoshima 890-0064, Japan

'Present address: Laboratory of Analytical and Bioanalytical Chemistry, School of Pharmaceutical Sciences, University of Shizuoka, Shizuoka 422-8526, Japan.

Adult T-cell leukemia/lymphoma (ATL) is an aggressive T-cell neoplasm caused by human T-cell leukemia virus type I (HTLV-I). Therapeutic interventions have not been associated with satisfactory outcomes. We showed that the porphyrin metabolic pathway preferentially accumulates the endogenous photosensitive metabolite, protoporphyrin IX (PpIX), in ATL, after a short-term culture with 5-aminolevulinic acid (ALA). PpIX accumulated 10-100-fold more in ATL leukemic cells when compared to healthy peripheral blood mononuclear cells (PBMCs). Patient specimens showed dynamic changes in flow cytometry profiles during the onset and progression of ATL. Furthermore, 98.7\% of ATL leukemic cell death in the ATL patient specimens could be induced with 10 min of visible light exposure, while $77.5 \%$ of normal PBMCs survived. Metabolomics analyses revealed that a specific stage of the metabolic pathway progressively deteriorated with HTLV-I infection and at the onset of ATL. Therefore, this method will be useful for diagnosing and identifying high-risk HTLV-I carriers and high-risk indolent ATL patients who appeared to have developed or are likely to develop the aggressive subtypes with single cell resolution. Photodynamic therapy in the circulatory system may be a potential treatment due to its highly-specific, non-invasive, safe, simultaneous, and repeatedly-treatable modalities.

\section{Preoperative localization of breast lesions: analysis of current techniques}

\section{Ray Cody Mayo}

The University of Texas MD Anderson Cancer Center, Houston, TX 77030, USA.

Image-guided preoperative localization of breast lesions is a relatively common procedure. This presentation describes the most common localization options available commercially - wire localization, radioactive seed localization, radiofrequency reflector localization, and magnetic seed localization - and outlines the advantages and disadvantages of each. This information may help radiologists, surgeons, pathologists, and hospital administration as they seek to add value and provide patient-centered care. 


\title{
18. Innovative technologies for cancer diagnosis and management metal-organic framework
} encapsulation for biospecimen and biotherapeutic preservation

\section{Jeremiah Morrissey ${ }^{1}$, Srikanth Singamaneni ${ }^{2}$}

${ }^{1}$ Department of Anesthesiology, Washington University in St. Louis, St. Louis, Missouri 63110, USA. ${ }^{2}$ Institute of Materials Science and Engineering and Department of Mechanical Engineering and Materials Science, Washington University in St. Louis, St. Louis, Missouri 63130, USA.

Background and aim: Handling, transport, and storage of biospecimens such as blood and urine without refrigeration are extremely challenging. This formidable challenge leads to an inevitable reliance on a "cold chain" for shipping, handling, and storage of biospecimens throughout the world. The cold chain requirement impedes biospecimen procurement from under-served populations and resource-limited settings where refrigeration and electricity are not reliable or even available.

Experimental procedure: Here, we introduce a universal biospecimen preservation approach based on nanoporous material encapsulation for preserving protein biomarkers in biofluids under non-refrigerated storage conditions. We used urinary neutrophil gelatinase-associated lipocalin and plasma CA-125 as the model protein biomarkers and measured their concentrations before and after encapsulation by enzymelinked immunosorbent assay (ELISA).

Results: We found that encapsulation in a zeolitic imidazolate framework-8 (ZIF-8), a nanoporous material, can preserve protein biomarkers in urine and plasma for weeks at room temperature and $40{ }^{\circ} \mathrm{C}$. The preservation efficacy for ELISA assay was greater than $85 \%$, comparable to freezing liquid samples at $-20{ }^{\circ} \mathrm{C}$. The protein biomarkers in the relevant biofluids were first encapsulated within the nanoporous ZIF-8 crystals, then dried on paper substrates via a dry spot sample collection method, and later reconstituted for analysis. This technology also preserves the biologic activity of insulin in liquid form for therapy.

Conclusion: This eco-friendly technology greatly improves biospecimen and biotherapeutic handling in resource-limited settings. The technology may be applicable to vaccine preservation, storage, and transport at ambient temperature. Overall, this environmentally-friendly and energy-efficient approach will alleviate huge financial and environmental burdens associated with "cold chain" facilities and extends biomedical research and treatment benefits to underserved populations from regions/populations currently inaccessible.

\section{Microrna-335-5p as a suppressor of metastasis and invasion in gastric cancer}

\author{
Iva Polakovicova ${ }^{1,2}$, Alejandra Sandoval-Borquez ${ }^{3,4}$, Nicolas Carrasco-Veliz ${ }^{1,2}$, Lorena Lobos- \\ González $^{3,4,5}$, Paulina González-Villarroel ${ }^{6}$, Alejandro Gottlieb-Riquelme ${ }^{6}$, Edison Salas- \\ Huenuleo ${ }^{4,7}$, Manuel Varas-Godoy ${ }^{8}$, Alejandro Corvalán ${ }^{1,2}$ \\ ${ }^{1}$ Laboratory of Oncology, Faculty of Medicine, Pontifícia Universidad Católica de Chile, Santiago 340, Chile. \\ ${ }^{2}$ Advanced Center for Chronic Diseases, Pontifícia Universidad Católica de Chile, Santiago 340, Chile. \\ ${ }^{3}$ Laboratory of Cellular Communication, Center for Studies on Exercise, Metabolism and Cancer (CEMC), \\ Institute of Biomedical Sciences (ICBM), Faculty of Medicine, Universidad de Chile, Santiago 1058, Chile. \\ ${ }^{4}$ Advanced Center for Chronic Diseases, Universidad de Chile, Santiago 1058, Chile. \\ ${ }^{5}$ Centro de Medicina Regenerativa, Faculty of Medicine, Universidad del Desarrollo, Santiago 456, Chile. \\ ${ }^{6}$ Escuela de Tecnología Médica, Faculty of Medicine, Universidad Andrés Bello, Santiago 239, Chile.
}


${ }^{7}$ Laboratory of Nanobiotechnology and Nanotoxicology, Facultad de Ciencias Químicas y Farmacéuticas, Universidad de Chile, Santiago 1058, Chile.

${ }^{8}$ Centro de Biología Celular y Biomedicina (CEBICEM) Campus Los Leones, Universidad San Sebastian, Santiago 1457, Chile.

Background and aim: Gastric cancer is the fifth most common cancer worldwide. It is mainly diagnosed via endoscopic examination, which is unsuitable for screening and patient follow-up. A deeper knowledge of its development and progression can contribute to discovering effective preventive strategies. To understand this complex process, we focus on microRNAs and exosomes and their metastatic and invasive potential on gastric cells.

Experimental procedure: We evaluated the expression of several candidate miRNAs in 38 gastric cancer tissues and 22 plasma samples from gastric cancer patients and compared them to adjacent not-tumor tissues and plasma from symptomatic patients without cancer, respectively. We performed an association analysis of the expression of microRNA-335-5p with clinicopathological features and survival curves. For in vivo study, we injected intravenously microRNA-335-5p-loaded exosomes into immunodeficient mice with intraperitoneal tumors.

Results: MicroRNA-335-5p is downregulated in advanced gastric cancer (GC) tissues relative to their paired non-tumor tissues. This downregulation is associated with worse survival rates of patients. We also demonstrated decreased levels of microRNA-335-5p in total plasma and exosomes isolated from plasma samples from GC patients, when compared to symptomatic patients without cancer. In our in vivo model of intraperitoneal carcinogenesis, we observed less metastasis but more necrosis in organs of mice with microRNA-335-5p-loaded exosomes and all mice lacked ascites.

Conclusion: MicroRNA-335-5p is downregulated in both types of gastric cancer samples. The difference in expression of this microRNA in plasma of gastric cancer patients versus patients without cancer is so profound that it can be considered as a possible candidate for non-invasive diagnosis of gastric cancer and the in vivo results may suggest a therapeutic role for miRNA-335.

\section{RANBP9 as potential target in non-small cell lung cancer}

\section{Vincenzo Coppola}

Department of Cancer Biology and Genetics, Ohio State University \& Comprehensive Cancer Center, Columbus, Ohio 43210, USA.

Non-Small Cell Lung Cancer (NSCLC) is by far the number one cause of cancer related death in the Western world. Despite the progress made with targeted therapies and immuno-checkpoint inhibitors, the vast majority of patients still undergo treatment with genotoxic drugs such as platinum-based compounds. Studies testing whether DNA damaging agents sensitize NSCLC tumors to targeted- or immuno-therapies are ongoing. However, only a better understanding of the mechanisms of the DNA damage response can lead to the validation of biomarkers predictive of response to genotoxic agents and the discovery of novel targets.

We found that overexpression of the scaffold protein Ran Binding Protein 9 (RANBP9) is pervasive in NSCLC. Most importantly, patients with higher levels of RANBP9 have a worst treatment outcome (Tessari et al $.^{[1]}, 2018$ ). Mechanistically, RANBP9 is not only a target (Matsuoka et al $.^{[2]}, 2007$ ) but also, 
surprisingly, an enhancer of the Ataxia telangiectasia mutated (ATM) kinase signaling (Palmieri et al. ${ }^{[3]}$, 2016). Indeed, the depletion of RANBP9 in NSCLC cells abates ATM activation and its downstream targets such as p53. Predictably, RANBP9 KO cells are more sensitive than controls to inhibition of the ataxia and telangiectasia-related kinase, but not ATM. Interestingly, the absence of RANBP9 renders cells more sensitive to drugs inhibiting Poly (ADP-ribose)-Polymerase (PARP) (Tessari et al. ${ }^{[1]}, 2018$ ).

We present results of our in vitro and in vivo investigation aimed at revealing the mechanisms responsible for increased sensitivity to specific genotoxic drugs when RANBP9 is absent. For this purpose, we generated human NSCLC cell lines and new mouse models of NSCLC in which endogenous RANBP9 can be specifically ablated in cancer cells or, alternatively, tagged with V5-HA for its unequivocal detection. Tumors of this latter group will enable proteomic studies to identify unknown RANBP9 interactions upon DNA damaging treatment.

\section{REFERENCES}

1. Tessari A, Parbhoo K, Pawlikowski M, Fassan M, Rulli E, et al. RANBP9 affects cancer cells response to genotoxic stress and its overexpression is associated with worse response to platinum in NSCLC patients. Oncogene 2018;37:6463-76.

2. Matsuoka S, Ballif BA, Smogorzewska A, McDonald ER, 3rd, Hurov KE, et al. ATM and ATR substrate analysis reveals extensive protein networks responsive to DNA damage. Science 2007; 316:1160-6.

3. Palmieri D, Scarpa M, Tessari A, Uka R, Amari F, et al. Ran Binding Protein 9 (RanBP9) is a novel mediator of cellular DNA damage response in lung cancer cells. Oncotarget 2016;7:18371-83.

\section{One carbon metabolic enzymes play important roles for cancer cells and cancer stem-like cells}

\section{Noriko Gotoh}

Cancer Research Institute, Kanazawa University, Ishikawa 920-1192, Japan.

Background and aim: Emerging evidence suggests that cancer stem-like cells (CSCs) are responsible for drug-resistant tumor recurrence ${ }^{[1-6]}$. The one-carbon $(1 \mathrm{C})$ metabolism incorporates carbons as building blocks of purine and pyrimidine that are used for DNA replication and RNA transcription. In the mitochondria, there are four major enzymes in $1 \mathrm{C}$ metabolism. These enzymes are strongly expressed in cancer cells, while it is scarcely expressed in normal cells. We investigated the role of MTHFD2 and MTHFD1L among them in cancer cells and CSCs.

Experimental procedure: We depleted expression of MTHFD2 and MTHFD1L in lung cancer cells and breast cancer cells by using small interfering RNA (siRNAs) or smal hairpin RNA (shRNAs). By using these cells, we examined cell proliferation and sphere-forming ability in vitro and in vivo. We also examined expression levels of stemness markers.

Results: We showed that MTHFD2 and MTHFD1L play important roles for cancer cell proliferation, stemlike properties, and drug resistance. Knockdown of MTHFD2 led to accumulation of 5-aminoimidazole carboxamide ribonucleotide (AICAR), an intermediate of the purine synthesis pathway, in association with reduced stem-like properties.

Conclusion: MTHFD2- or MTHFD1L-mediated mitochondrial 1C metabolism appears critical for survival of CSCs through consumption of AICAR, leading to depletion of the intracellular pool of AICAR. Because CSCs are dependent on MTHFD2 and MTHFD1L, therapies targeting MTHFD2 may eradicate tumors. 


\section{REFERENCES}

1. Takahiko M, Noriko G. Drug resistance mechanisms of cancer stem-like cells and their therapeutic potential as drug targets. Cancer Drug Resist 2019;2:457-70.

2. Tominaga K, Minato H, Murayama T, Sasahara A, Nishimura T, et al. Semaphorin signaling via MICAL3 induces symmetric cell division to expand breast cancer stem-like cells. Proc Natl Acad Sci U S A 2019;116:625-30.

3. Nishimura T, Nakata A, Chen X, Nishi K, Meguro-Horike M, et al. Cancer stem-like properties and gefitinib resistance are dependent on purine synthetic metabolism mediated by the mitochondrial enzyme MTHFD2. Oncogene 2019;38:2464-81.

4. Tominaga K, Shimamura T, Kimura N, Murayama T, Matsubara D, et al. Addiction to the IGF2-ID1-IGF2 circuit for maintenance of the breast cancer stem-like cells. Oncogene 2017;36:1276-86.

5. Murayama T, Nakaoku T, Enari M, Nishimura T, Tominaga K, et al. Oncogenic fusion gene CD74-NRG1 confers cancer stem cell-like properties in lung cancer through a IGF2 autocrine/paracrine circuit. Cancer Res 2016;76:974-83.

6. Nakata A, Yoshida R, Yamaguchi R, Yamauchi M, Tamada Y, et al. Elevated $\beta$-catenin pathway as a novel target for patients with resistance to EGF receptor targeting drugs. Sci Rep 2015;5:13076.

\section{Differential roles of the redox-sensitive transcription factor, Nrf2 in multistage carcinogenesis}

\section{Young-Joon Surh}

Tumor Microenvironment Global Core Research Center, College of Pharmacy and Department of Molecular Medicine and Biopharmaceutical Sciences, Graduate School of Convergence Science and Technology, Seoul National University, Seoul 08826, South Korea.

Background and aim: Nuclear factor E2-related factor 2 (Nrf2) is a redox-sensitive transcription factor regulating the expression of a battery of genes encoding antioxidant and carcinogen detoxifying enzymes. In contrast to its tumor suppressive functions in normal cells, Nrf2 facilitates tumor growth and progression through metabolic reprograming in some cancer cells. Our previous study has demonstrated that 15-deoxy- $\Delta^{12,14}$-prostaglandin $\mathrm{J}_{2}$ and 4-hydroxyestradiol induce overactivation of Nrf2 and consequently overexpression of its target protein, heme oxygenase-1 (HO-1), in human breast cancer cells.

Experimental procedure: In this study, we investigated the involvement of Nrf2 in experimentally induced hepatocarcinogenesis by utilizing Nrf2 null mice as well as wild type animals. The liver tumor was induced by intraperitoneal injection of diethylnitrosamine (DEN). The expression of Nrf2 and its target genes and proteins were measured by RT-PCR and Western blot analyses. The cell proliferation was determined by immunohistochemical analysis of Proliferating Cell Nuclear Antigen expression.

Results: Nrf2 expression, nuclear translocation, and transcriptional activity were enhanced in liver tumors. Overactivated Nrf2 was required for hepatoma growth in DEN-induced HCC. Following DEN treatment, Nrf2 genetic disruption reduced expression of pentose phosphate pathway-related enzymes, the depletion of which has been associated with an amelioration of HCC incidence. Nrf2-deficient mice resisted DENinduced hepatocarcinogenesis.

Conclusion: The cellular stress response or cytoprotective signaling mediated via the Nrf2 is often hijacked by cancer cells. This may facilitate the remodeling of the tumor microenvironment, making it advantageous for the autonomic growth of cancer cells, metastasis, angiogenesis, tolerance to anticancer therapy, and selfrenewal activity of stem-like cells. Notably, Nrf2 overactivation upregulates antioxidant gene expression in breast cancer stem cells, which contributes to the manifestation and maintenance of stemness. 


\title{
23. The importance of sequential mutations in pancreatic tumorigenesis
}

\section{Gloria Su}

Columbia University Irving Medical Center, New York, NY 10032, USA.

Background and aim: Genetically engineered animal models (GEMMs) are established robust platforms for exploring the molecular mechanisms underlying the progression of pancreatic precancerous lesions to invasive PDA (pancreatic ductal adenocarcinoma). For example, using Pdx1-Cre to activate mutant $\operatorname{Kras}^{G 12 D}$ allele in the pancreas induces full spectrum of premalignant PanIN (pancreatic intraepithelial neoplasias) lesions that can eventually progress to invasive PDA (reviewed in $^{[1]}$ ). We and others have reported that concomitant inactivation of the tumor suppressors $p 16, p 19, p 53$, or TGF- $\beta$ receptor type 2 $(T g f \beta R 2)$ can synergize with oncogenic $\mathrm{Kras}^{\mathrm{G}}{ }^{212}$ in promoting the progression of the non-invasive PanINs to invasive cancer in $v i v o^{[1,2]}$. In contrast, the inactivation of Smad4 or Acvr1b in the context of mutant $\mathrm{Kras}^{\mathrm{G}}{ }^{2 \mathrm{D}}$ preferentially promotes the development of pancreatic IPMNs (intraductal papillary mucinous neoplasms) but not PanINs ${ }^{[1,3]}$. Collectively, these data suggest that the order in which tumor-suppressor genes are inactivated may influence the development of pancreatic tumor subtypes. To further investigate the importance of sequential mutations in pancreatic tumorigenesis, we generated double heterozygous Smad ${ }^{\text {flox } /} ;$; $16^{+/-} ;$LSL-KRAS G12D;Pdx1-Cre GEMM and asked how spontaneous inactivation of the second allele might impact the development pancreatic precancerous lesions.

Experimental procedure: Smadt ${ }^{\mathrm{flox} / \mathrm{t}} ;$ p1 $6^{+/-} ; \mathrm{LSL}-\mathrm{KRAS}$ G12D;Pdx1-Cre mice were examined and characterized in comparison to $p 16^{+/-} ; L S L-K R A S$ G12D;Pdx1-Cre and Smad4 ${ }^{\text {flox/+ }} ;$ p $16^{+/-} ; P d x 1-C r e$ GEMMs.

Results: Smad4 ${ }^{\mathrm{flox} /+} ; p 16^{+/-} ; L S L-K R A S$ G12D;Pdx1-Cre mice shared similar medium survival and tumor progression to $p 16^{+/} ; L S L-K R A S$ G12D;Pdx1-Cre mice (PanIN to PDA). Molecular analyses showed that biallelic inactivation only occurred at the $p 16$ locus in the PanINs and PDA from Smad4 ${ }^{\text {flox } /+} ; p 16^{+/-} ; L S L-$ KRAS G12D;Pdx1-Cre GEMM.

Conclusion: Our results support the previous observations that the sequential inactivation of tumorsuppressor genes in the context of oncogenic $\mathrm{Kras}^{G 12 \mathrm{D}}$ can dictate the development of pancreatic precancerous lesions. More importantly, the sequential mutations observed in mice mirror those detected in human patient specimens and thus illustrating that the order of genetic mutations is as critical as the mutated genes themselves in influencing tumor development and progression.

\section{REFERENCES}

1. Qiu W, Su GH. Challenges and advances in mouse modeling for human pancreatic tumorigenesis and metastasis. Cancer Metastasis Rev 2013;32:83-107.

2. Qiu w, Sahin F, Iacobuzio-Donahue CA, Garcia-Carracedo D, Wang WM, et al. Disruption of p16 and activation of Kras in pancreas increases ductal adenocarcinoma formation and metastasis in vivo. Oncotarget 2011;2:862-73.

3. Qiu W, Tang SM, Lee S, Turk AT, Sireci AN, et al. Loss of activin receptor type 1B promotes development of intraductal papillary mucinous neoplasms in mice with activated KRAS. Gastroenterology 2016;150:218-28.e12.

\section{Cyr61 promotes tip cell activity through VEGFR2-Hippo pathway in tumor angiogenesis}

\author{
Sarala Manandhar ${ }^{1}$, Uttam Ojha ${ }^{1}$, Hyeonha Jang ${ }^{1}$, Sun-Hee Lee ${ }^{1}$, Soo-Hyun Yoon ${ }^{1}$, Li Kang ${ }^{1}$, \\ Myo-Hyeon Park', You Mie Lee ${ }^{1,2}$
}

${ }^{1}$ BK21 Plus KNU Multi-Omics based Creative Drug Research Team.

${ }^{2}$ Research Institute of Pharmaceutical Sciences, College of Pharmacy, Kyungpook National University, Daegu 41566, Republic of Korea. 
Cyr61 stimulates active angiogenesis in various tumors, although the mechanism is unknown. Here, we report that Cyr61 enhances the activity of tip cells during angiogenesis by regulating VEGFR2Hippo pathway. Microvessel networks and directional vascular cell migration patterns were deformed in Cyr61-knockdown zebrafish embryos. Moreover, Cyr61 promoted the endothelial sprouting activity in angiogenesis. Cyr61 induced the interaction of integrin $\alpha v \beta 3$ with VEGFR2, which activated downstream MAPK/PI3K signaling pathways, YAP/TAZ, and Rho effector mDia1 to enhance tip cell activity and Cyr61 itself. Integrin $\alpha v \beta 3$ inhibitor repressed tip cell number and sprouting in postnatal retinas from endothelial cell-specific Cyr61 transgenic mice (VE-Cadherin:Cyr61), and allograft tumors in Cyr61 transgenic mice also showed hyperactive vascular sprouting. Cancer patients with high Cyr61 expression have poor survival outcomes and positive correlation with integrin $\alpha v \beta 3$ and high YAP/TAZ. Thus, our data underscore the positive feedback regulation of tip cells by Cyr61 through integrin $\alpha$ v $\beta 3 /$ VEGFR2 and YAP/TAZ activity, suggesting a promising therapeutic intervention for pathological angiogenesis.

\section{Silibinin targets bone morphogenic protein $\mathbf{2}$ in its efficacy against ultraviolet $B$ radiation- induced promotion/progression of microscopic basal cell carcinoma formation}

\section{Rajesh Agarwal, Chapla Agarwal}

Department of Pharmaceutical Sciences Skaggs School of Pharmacy and Pharmaceutical Sciences, University of Colorado Anschutz Medical Campus, Aurora, CO 80045, USA.

Background and aim: Non-melanoma skin cancers (NMSCs) account for about half of all malignancies diagnosed annually in the United States. Around $80 \%$ of NMSCs are basal cell carcinoma (BCC) and $20 \%$ are squamous cell carcinoma (SCC). Whereas the efficacy of several chemopreventive agents has been examined and reported against both BCC and SCC, a majority of these studies have focused on the test agent's activity in a long-term setting to determine the number of tumors formed. Notably, the studies evaluating the efficacy of chemopreventive agents during early stage(s) of BCC development are lacking. Accordingly, utilizing the well-established patched (Ptch)+/- mouse model of ultraviolet $b$ (UVB)-induced BCC formation, we excised skin samples from UVB exposed mice prior to tumor formation to study the promotion/progression of BCC and to determine the target(s) of silibinin, a well-known skin cancer (SCC) chemopreventive agent, in BCC tumor growth inhibition.

Experimental procedure: We used a multifactor approach: (1) long-term ultraviolet B radiation-induced mouse skin tumorigenesis in Ptch heterozygous mice focusing on BCC; (2) investigating and quantifying expression of molecular regulators and cyclobutane pyrimidine dimers by immunohistochemistry and/or immunoblotting; and (3) real-time PCR with mouse signal transduction pathway finder PCR array.

Results: At as early as one month, we found that UVB exposure significantly increased the number of mast cells in Ptch $+/-$ mice by about $48 \%(P<0.05)$, which was completely inhibited (to control levels) by silibinin topical treatments. In Ptch $+/+$ mice, which do not develop BCC tumors, we did not observe any increase in mast cells following UVB exposure, suggesting this could be a specific pathway in the development of BCC. To decipher the molecular mechanism of these findings, we performed a PCR profiler array analysis of several genes involved in signal transduction pathways which showed strong differences between Ptch $+/+$ and Ptch $+/-$ mice that were unexposed, UVB irradiated, and silibinin treated. Most notably, following UVB exposure for one month, in Ptch + /- mice, the expression of Bone Morphogenetic Protein 2 (BMP-2), Hairy/enhancer-of-split related with YRPW motif 1 (Hey1), and Inhibitor of DNA binding 1 (Id1) was significantly upregulated when compared to Ptch $+/+$ mice. Additional studies focusing on BMP-2 found that silibinin strongly inhibits UVB-induced expression of BMP-2 in Ptch+/- mouse 
skin. Consistent with these results, we also found that silibinin strongly attenuates UVB-induced BMP-2 expression and DNA damage in Ptch $+/-$ mouse skin ex vivo. Regarding BCC formation, silibinin treatment inhibited UVB-induced microscopic BCC formation in Ptch+/- mice; microscopic tumor number and size were reduced by $73 \%$ and $84 \%$, respectively. Together, our results suggest a possible role of BMP- 2 in early stages of BCC development and that silibinin plausibly acts through BMP-2 to inhibit microscopic BCC formation.

Conclusion: Our current findings in BCC model, together with previous studies in SCC model, suggest that silibinin could be a multi-target agent capable of being a chemopreventive agent for both types of NMSCs.

\section{A novel sulforaphane-regulated gene network in prevention of breast cancer-induced osteolytic bone resorption}

\section{Shivendra V. Singh}

Department of Pharmacology \& Chemical Biology, and UPMC Hillman Cancer Center, University of Pittsburgh, Pittsburgh, PA 15260, USA.

Bone is the most preferred site for colonization of metastatic breast cancer cells for each subtype of the disease. The standard of therapeutic care for breast cancer patients with bone metastasis include bisphosphonates (e.g., zoledronic acid), which have poor oral bioavailability, and a humanized antibody (denosumab). However, these therapies are palliative and a subset of patients still develop new bone lesions and/or experience serious adverse effects. Therefore, a safe and orally bioavailable intervention for prevention/therapy of osteolytic bone resorption is still a clinically unmet need. This study demonstrates prevention of breast cancer-induced bone resorption by a small molecule (sulforaphane, SFN) that is safe clinically and orally bioavailable. in vitro osteoclast differentiation was inhibited in a dose-dependent manner upon addition of conditioned media from SFN-treated breast cancer cells representative of different subtypes. Targeted microarray coupled with interrogation of TCGA dataset revealed a novel SFNregulated gene signature involving cross-regulation of runt-related transcription factor 2 (RUNX2) and nuclear factor- $\mathrm{\kappa B}$ and their downstream effectors. Both RUNX2 and p65/p50 expression were higher in human breast cancer tissues compared to normal mammary tissue. RUNX2 was recruited at the promotor of NFKB1. Inhibition of osteoclast differentiation by SFN was augmented by doxycycline-inducible stable knockdown of RUNX2. Oral SFN administration significantly increased the percentage of bone volume/ total volume of affected bones in the intracardiac MDA-MB-231-Luc model, indicating in vivo suppression of osteolytic bone resorption by SFN. These results indicate that SFN is a novel inhibitor of breast cancerinduced osteolytic bone resorption in vitro and in vivo. These findings necessitate clinical investigations to determine the effect of SFN administration on osteolytic bone resorption in women with metastatic breast cancer. This study was supported by grant CA225716 awarded by the National Cancer Institute.

\section{Targeting of TM4SF5-mediated regulation of metabolic functions to overcome hepatic cancer}

\section{Jung Weon Lee}

College of Pharmacy, Seoul National University, Seoul 08826, South Korea.

Background and aim: Liver is an organ that can metabolize diverse nutrients and its cancers show arginine auxotroph, which involves arginine delivery from extracellular diet sources and lysosomal protein 
degradation products. Among the genes involved in hepatic cancer, transmembrane $4 \mathrm{~L}$ six family member 5 (TM4SF5), which is structurally similar to the tetraspanins with 4 transmembrane domains, is shown to be highly expressed in cancerous liver tissues and correlated with many hepatic metabolism genes. However, it is unknown whether and how TM4SF5 is involved in arginine metabolism in livers.

Experimental procedure: We examined whether TM4SF5 expression can be involved in the mTORC1 signaling pathway, since mTORC1 signaling is a central hub of various cellular metabolism processes.

Results: First, we found shuttling of TM4SF5 between plasma membrane and lysosomal (late endosomal) membrane, depending on availability of amino acids. Further, upon resupply of arginine to argininestarved cells, lysosomal TM4SF5 was associated with mTOR, leading to an increased S6K phosphorylation. The association between TM4SF5 and mammalian target of rapamycin (mTOR) appeared to require the C-terminal regions of TM4SF5 and kinase activity of mTOR. In addition, an endosomal arginine transporter (SLC38A9) and a cytosolic arginine sensor (Castor1) were found to be associated with $\mathrm{TM} 4 \mathrm{SF} 5$, indicating TM4SF5 as an arginine sensor on late endosomal (lysosomal) membrane. Interestingly, the association of Castor 1 with TM4SF5 was negatively regulated by $L$-arginine, but concomitantly the association between mTOR and TM4SF5 increased. Furthermore, certain residues in the extracellular loop 2 of TM4SF5 bound to arginine. Thus, association of TM4SF5 with mTOR, SLC38A9, and arginine on lysosomal membrane might allow $\mathrm{TM} 4 \mathrm{SF} 5$ to propagate arginine response to $\mathrm{mTORC} 1$ by directly sensing arginine in the lysosome as well as to elevate the cytosolic arginine pool for cellular homeostasis.

Conclusion: Therefore, these observations suggest TM4SF5 as an arginine sensor on late endosomal membrane and as a promising therapeutic target candidate for the arginine auxotroph of hepatic cancers.

\title{
28. Targeting cancer stem cells in malignant mesothelioma
}

\section{Richard L. Eckert ${ }^{1,2,4}$, Gautam Adhikary ${ }^{1}$, Daniel Grun', Joseph S. Friedberg ${ }^{3,4}$, Wen Xu', Jeffrey Keillor $^{5}$, Sivaveera Kandasamy ${ }^{3}$, H. Richard Alexander ${ }^{6}$}

\author{
${ }^{1}$ Department of Biochemistry and Molecular Biology, University of Maryland School of Medicine, Baltimore, \\ MD 21201, USA. \\ ${ }^{2}$ Department of Dermatology, University of Maryland School of Medicine, Baltimore, MD 21201, USA. \\ ${ }^{3}$ Department of Surgery and Division of General and Surgical Oncology, University of Maryland School of \\ Medicine, Baltimore, MD 21201, USA. \\ ${ }^{4}$ Greenebaum Comprehensive Cancer Center, University of Maryland School of Medicine, Baltimore, MD \\ 21201, USA. \\ ${ }^{5}$ Department of Surgery, Robert Wood Johnson Medical School, New Brunswick, New Jersey, NJ 08901, USA. \\ ${ }^{6}$ Department of Chemistry, University of Ottawa, Ottawa, ON K1N 6N5, Canada.
}

Background and aim: Mesothelioma is an aggressive, treatment resistant, and fatal cancer of the mesothelial lining of the pleural and peritoneal cavities that is initiated by exposure to asbestos or nanotubes. Surgical reduction and chemotherapy are first line treatments, but recurrence of highly aggressive and drug-resistant disease is common. Disease recurrence is associated with expansion of mesothelioma cancer stem cells (MCS cells). Thus, new treatments are needed for this disease that target the cancer stem cell population.

Experimental procedure: We used genetic gene expression and knockdown approaches, signaling studies, xenograft tumor studies, transcriptome analysis, and protein structure studies to characterize the role of the transglutaminase 2 (TG2) cancer stem cell survival factor in enhancing MSC cell survival and function. 
Results: We showed that tissue transglutaminase (TG2), a cancer stem cell survival and drug-resistance protein, is highly enriched in human mesothelioma tumors and mesothelioma cancer stem cells (MCS cells) and drives MCS cell spheroid formation, invasion, and migration. TG2 knockdown or TG2 inhibitor treatment reduces MCS cell survival, spheroid formation, Matrigel invasion, migration, and tumor formation. These are important observations as MCS cells comprise a highly aggressive subpopulation of tumor that forms rapidly growing and aggressive tumors. In addition, transcriptome analysis reveals that TG2 loss is associated with reduced levels of mRNA encoding a wide range of cancer stem cell and epithelial-mesenchymal transition proteins, and that TG2 knockdown reduces expression of transcripts and proteins encoding pro-cancer matrix proteins including collagens COL1A 2 and COL3A1 that are involved in metastasis. Mesothelin, a mesothelioma cell-specific MCS cell survival protein and attachment factor, is also reduced in TG2 knockdown cells.

Conclusions: These studies indicate that TG2 is highly overexpressed in MCS cells and drives the cancer stem cell phenotype to enhance MCS cell stemness, survival, and invasion, and suggests that TG2 is an important candidate mesothelioma cancer stem cell therapy target.

\section{Forward genetics to discover tumor suppressor in colorectal cancer}

\section{Tao Lu}

Department of Pharmacology and Toxicology, Indiana University School of Medicine, 635 Barnhill Drive, Indianapolis, IN 46202, USA.

The nuclear factor $\kappa \mathrm{B}(\mathrm{NF}-\kappa \mathrm{B})$ plays pivotal roles in inflammatory and immune responses and in cancer. Therefore, understanding its regulation holds great promise for disease therapy. Using validationbased insertional mutagenesis, a powerful technique established by us, we discovered a novel negative regulator of NF- $\mathrm{B}$ (named NNRN1) in colorectal cancer (CRC). We showed that NNRN1 overexpression downregulated the expression of NF- $\mathrm{B}$-dependent genes, many of which are related to cancer. Additionally, compared to the vector control group, overexpression of NNRN1 in HEK293 cells or CRC HT29, DLD1, and HCT116 cells dramatically reduced NF- $\kappa$ B activity, cellular proliferation, anchorageindependent growth, and migratory ability in vitro, and, unsurprisingly, significantly decreased xenograft tumor growth in vivo. In contrast, shNNRN1 knockdown cells showed the opposite effect. Furthermore, co-immunoprecipitation (Co-IP) experiment confirmed that NNRN1 may form a complex with the p65 subunit of NF- $\mathrm{KB}$. Importantly, immunohistochemistry data exhibited much lower NNRN1 expression level in CRC patient tumor tissues compared to normal tissues, indicating that NNRN1 may function as a tumor suppressor in CRC. To conclude, our findings for the first time uncovered the negative regulatory function of NNRN1 in NF- $\mathrm{B}$ signaling, and present NNRN1 as an innovative therapeutic target in CRC treatment.

\section{Immunogenic cell death in myeloid leukemia}

\section{Marc Diederich}

College of Pharmacy, Seoul National University, Seoul 08826, South Korea.

Background and aim: We investigated the effect of pharmacologically active compounds that act as immunoadjuvants able to trigger a cancer stress response and release of damage-associated molecular patterns (DAMPs) in myeloid leukemia ${ }^{[1]}$. These processes result in a chemotherapeutic response with 
a potent immune-mediating reaction. Several parameters determine whether a compound can act as an immunogenic cell death (ICD) inducer including the nature of the inducer, the premortem stress pathways, the cell death pathways, the intrinsic antigenicity of the cell, and the potency and availability of an immune cell response ${ }^{[2]}$.

Experimental procedure: We used a multifactor approach: (1) detecting endoplasmic reticulum (ER) stress markers; (2) investigating and quantifying caspase-dependent or independent cell death; (3) measuring the release of danger associated molecular patterns; (4) quantifying phagocytosis of compound-treated cells by both murine and human monocyte-derived macrophages; (5) performing colony formation assays and in vivo zebrafish xenografts; and (6) performing vaccination assays with immunocompetent mice.

Results: We identified ICD-inducing capacities of old (coumarinics) and novel (stemphol and cardiac glycoside UNBS1450) inducers of immunogenic cell death together with venetoclax and experimental $\mathrm{BH} 3$ mimetics. We detected their capacity to trigger synergistic cell death in myeloid leukemia in an attempt to overcome apoptosis-resistant myeloid leukemia alone or in combination with other chemotherapeutic compounds.

Conclusion: The identification of hallmarks of ICD is important in determining the prognostic biomarkers for new therapeutic approaches and combination treatments ${ }^{\left[{ }^{[3]}\right.}$. In myeloid leukemia, combination treatments of ICD-inducing pharmacological agents ${ }^{[4]}$ with Venetoclax showed positive synergistic effects ${ }^{[5]}$ allowing to confer immunogenicity to otherwise cytotoxic non-immunogenic treatments.

\title{
REFERENCES
}

1. Radogna F, Diederich M. Stress-induced cellular responses in immunogenic cell death: Implications for cancer immunotherapy. Biochem Pharmacol 2018;153:12-23.

2. Mazumder A, Lee JY, Talhi O, Cerella C, Chateauvieux S, et al. Hydroxycoumarin OT-55 kills CML cells alone or in synergy with imatinib or Synribo: Involvement of ER stress and DAMP release. Cancer Lett 2018;438:197-218.

3. Ji S, Lee JY, Schrör J, Mazumder A, Jang DM, et al. The dialkyl resorcinol stemphol disrupts calcium homeostasis to trigger programmed immunogenic necrosis in cancer. Cancer Lett 2018;416:109-23.

4. Diederich M, Muller F, Cerella C. Cardiac glycosides: From molecular targets to immunogenic cell death. Biochem Pharmacol 2017;125:1-11.

5. Cerella C, Gaigneaux A, Mazumder A, Lee JY, Saland E, et al. Bcl-2 protein family expression pattern determines synergistic pro-apoptotic effects of BH3 mimetics with hemisynthetic cardiac glycoside UNBS1450 in acute myeloid leukemia. Leukemia 2017;31:755-9.

\section{The role of autophagy in inflammatory cytokines-induced epithelial to mesenchymal}

\section{transition in cancer}

\section{Chiara Vidoni ${ }^{1}$, Alessandra Ferraresi ${ }^{1}$, Eleonora Secomandi ${ }^{1}$, Letizia Vallino ${ }^{1}$, Suyanee Thongchot ${ }^{1}$, Danny Dhanasekaran ${ }^{2}$, Ciro Isidoro $^{1}$}

\author{
${ }^{1}$ Department of Health Sciences, Università del Piemonte Orientale, Novara 28100, Italy. \\ ${ }^{2}$ Stephenson Cancer Center, Oklahoma University Health Science Center, Oklahoma City, OK 73104, USA.
}

The peculiar hallmark distinguishing malignant from benign tumors is the ability of the former to invade the extracellular matrix and metastasize to near and distant organs. This process implies an epigenetic change in the expression of genes that leads to a reversible phenotypic change of the cancer cells from epithelial-like to mesenchymal-type known as epithelial-to-mesenchymal transition (EMT). The tumor microenvironment plays a pivotal role in this process, the major players being the pro-inflammatory cytokines IL- 6 and IL-8 released by cancer associated fibroblasts, immune cells (M2 macrophages), and cancer cells themselves. 
Autophagy, a lysosome-driven catabolic process for degradation of self-constituents, participates in the stress response for maintaining cell homeostasis. It has been shown that autophagy is down-regulated during cell locomotion, while it is induced when cells arrest their migration.

We found that pro-inflammatory cytokines promote cancer cell migration following down-regulation of autophagy in the migratory cells. We also investigated at the molecular level the mechanisms through which the cytokines modulate autophagy.

Our data highlight the role of autophagy in cancer cell EMT and migration, offering opportunities for therapeutic interventions to prevent invasion and metastasis.

\section{Vasculogenic mimicry in glioblastoma and melanoma}

\section{Luca Colucci-D’Amato, Maria Teresa Gentile, Olga Pastorino}

Laboratory of Molecular and Cellular Neuropathology, Department of Environmental, Biological and Pharmaceutical Science and Technology, University of Campania "L. Vanvitelli", Caserta 81100, Italy.

Background and aim: Neo-angiogenesis is the most studied mechanism of vascularization in tumors and refers to sprouting of new blood vessels from pre-existing ones and it may be inhibited by natural compounds. Vasculogenic mimicry (VM) is an alternative mechanisms of tumor vascularization providing a means by which some tumors can escape anti-angiogenetic therapy. VM occurs in glioblastoma (GBM) and melanoma, both tumors of neuroepithelial derivation. We investigated the role of REST/NRSF gene in the pathophysiology of VM as well as the effects of compounds such as the histone deacetylase inhibitors (HDACis) to interfere with VM.

Experimental procedure: To measure tube formation, cell migration, and invasion, we used: in vitro tube formation assay on Matrigel, Boyden chamber migration assay, wound healing assay, invasion test, and real-time migration monitoring. To measure cell viability, we used: MTT and trypan blue exclusion test. To transfect cells, we used: lipofectamine standard protocol.

Results: We analyzed a number of GBM and melanoma cell lines. We found that the expression of REST parallels the ability to migrate and to form tubes on Matrigel. Upon genetic or chemical down-regulation of REST (via siRNA or dominant negative mutant or HDACi), we observed a decrease in migration ability as well as tube formation. Finally, we found that different histone deacetylase inhibitors impair vasculogenic mimicry from glioblastoma cells.

Conclusions: Our findings show that REST/NRSF gene is an important molecular player in the pathophysiology of vasculogenic mimicry in GBM and melanoma and that HDAC inhibitors alone can impair the formation of tubes from GBM cells.

\section{Mouse tumor susceptibility alleles identify pathways for intervention in multiple myeloma}

\section{Beverly A. Mock}

Laboratory of Cancer Biology \& Genetics, Center for Cancer Research, National Cancer Institute, National Institutes of Health, Bethesda, MD 20892, USA. 
Background and aim: Multiple Myeloma (MM) is a clonal proliferation of neoplastic plasma cells in the bone marrow. Despite recent therapeutic advances, drug resistance and MM progression is common. Mouse plasma cell tumors model these antibody producing neoplasms. Long-term genetic studies utilizing backcross, and congenic strain analyses coupled with positional cloning strategies and functional studies identified Cdkn2a, Mtor, and Mndal as plasmacytoma susceptibility genes. Tumor incidence data in congenic strains carrying resistance alleles of Cdkn2a and Mtor led us to hypothesize that drug combinations affecting these pathways are likely to have an additive, if not synergistic, effect in inhibiting tumor cell growth.

Experimental procedure: Drug combination [mTOR and histone deacetylases (HDAC) inhibitors] activity and synergy were measured in B cell neoplasms and NCI-60 cell lines. in vivo activity was assessed in xenograft experiments. Co-expression network analyses of microarray data from in vitro drug treatment delineated the cooperative mTORi/HDACi transcriptional response. Selectivity of the response for genes differentially regulated in MM was determined by GSEA of datasets from healthy controls and MM patients. The combination's potential clinical utility was evaluated by developing a multivariate survival prediction model from the response signature in a MM patient dataset. Functional enrichment and transcription factor activity testing of the response signature delineated the combination's biological activities.

Results: The combination was active and synergistic in $90 \%$ of cell lines and controlled in vivo tumor growth for 12 weeks. Combination response signature genes were correlated with improved survival and the signature was functionally enriched for cell cycle, apoptosis, antigen presentation, and DNA damage response. The combination is predicted to repress oncogenic factors and activate tumor suppressors (RB1 and $\mathrm{CDKN} 2 \mathrm{~A}$ ).

Conclusion: The traditional and novel systems-level genomic approaches used to assess combination activity, disease specificity, and clinical potential demonstrate the efficacy of combined mTORi/HDACi, and warrant further investigation in clinical trials.

\section{4. tRNA-derived fragment AS-tDR-007333 promotes cell proliferation in NSCLC through interacting with HSPB-1}

\section{Wenyan Yang ${ }^{1}$, Lin Yang ${ }^{2}$, Qihan He ${ }^{1}$, Peikun Ding ${ }^{2}$, Zheng Wang ${ }^{2}$, Lijuan Ling ${ }^{2}$, Yi Song ${ }^{1}$, Rihong Zhai ${ }^{1}$}

${ }^{1}$ Shenzhen University School of Medicine, Shenzhen 518060, Guangdong, China.

${ }^{2}$ Shenzhen People's Hospital, Shenzhen 518055, Guangdong, China.

Background: tRNA derived-fragments (tRFs) comprise a new class of non-coding small-molecule RNA. Recent studies suggest that tRFs are involved in the development and progress of several cancers. However, the impact of tRFs in non-small cell lung cancer (NSCLC) remains elusive.

Methods: NSCLC-related tRFs were determined by RNA-seq. Expression of tRFs in tumor tissues, plasma, and NSCLC cell lines was analyzed with qRT-PCR. The effect of tRFs on NSCLC malignancy was evaluated in vitro by loss- and gain-of-function assays. RNA-seq was conducted to screen for the target genes of tRFs. The mechanism of action of tRFs was explored with RNA pulldown, RNA immunoprecipitation (RIP), and qRT-PCR. 
Results: RNA-seq identified seven differentially expressed tRFs between pre-and postoperative plasmas in patients with NSCLC. Among them, the expression of a novel tRF termed AS-tDR-007333 was significantly upregulated in preoperative plasma, NSCLC tissues, and NSCLC cell lines. Overexpression of AS-tDR-007333 promoted NSCLC cell (PC9, HCC827, and A549) proliferation, while knockdown of AS-tDR-007333 inhibited cell growth. RNA-seq showed that up-regulation of AS-tDR-007333 led to the activation of oncogenes such as MED29, AL049829.1, SCHIP1, SAMD12, MRFAP1, and SHISA5. RNA pulldown and RIP analyses revealed that AS-tDR-007333 can bind directly with heat shock protein beta-1 (HSPB-1). Rescue assays demonstrated that HSPB1 was involved in AS-tDR-007333 mediated NSCLC cell proliferation.

Conclusion: Our study reveals an oncogenic role of AS-tDR-007333 in NSCLC, suggesting that it may be a novel target for diagnosis and the treatment of NSCLC.

\section{Breast tumor-on-chip}

\section{Subia Bano}

Elvesys Microfluidics Innovation Centre Paris, Paris 75011, France.

Background and aim: Breast cancer is the most common invasive cancer among women. There are several chemotherapeutic and radiotherapeutic approaches available but they have certain limitations. Over the past few years, improved understanding of the microenvironment heterogeneity of breast cancer has allowed the development of more effective treatment strategies. However, researchers have still not been able to recapitulate the entire tumor microenvironment to study tumor progression and invasion. In this way, more complex 3D in vitro cancer models have been developed. These $3 \mathrm{D}$ tumor models still lack the cell-cell and cell-tissue interactions and more balanced interstitial fluidic flow that are present within living systems. Furthermore, mimicking different physiological conditions and collecting samples from tumor microenvironment are also difficult. In this direction, the breast tumor-on-chip model has emerged as an alternative system to study the tumor microenvironment and decipher its role in metastasis. In this work, a microfluidics system was integrated into a $3 \mathrm{D}$ breast tumor to bridge the gap between $2 \mathrm{D}$ and animal model effectively and evaluate the efficacy of anti-cancerous drugs. These microfluidic systems contain small chambers for cell culture, which enable control over local gradients and the ability to maintain the interstitial fluidic flow of the local breast tumor microenvironment.

Experimental procedure: In this work, the multi-compartment microfluidics platform was generated by designing a specific PDMS chip with three channels that are separated by specific barriers $(50 \mu \mathrm{m})$. The cancerous and fibroblast cells (cocultures) were suspended with collagen hydrogel and loaded into the central channel and one of the side channels was used to grow the endothelial cells to make this system vascularized. The barriers inside the chips allowed exchanging signaling molecules.

Results: The cancer cells in the presence of fibroblast cells grew well in these microchannels, which was confirmed with live/dead assay. At this stage, we have obtained the preliminary data and are still working in this area.

Conclusion: Integration of microfluidics system into breast tumor will add another toolset that can make a more efficient testing platform for the current therapeutic development pipeline. 


\title{
36. Giant mediastinal mixed germ cell tumor, a rare case report and review of literature
}

\section{Abdulrahman Hakami}

Jazan University, Jazan 45142, KSA.

Introduction: Germ cell tumors are relatively rare, embryologically-derived from reproductive cells, and usually arise in the gonads. Mediastinal germ cell tumors are estimated to be about 1\%-3\% of all germ cell tumors and are generally seen in the anterior mediastinum, while metastatic lesions are mostly seen in the posterior mediastinum. The most aggressive germ cell tumor subtypes are choriocarcinoma, embryonal carcinoma, and yolk-sac tumors. Seminomas only rarely spread distantly. The presentations vary, ranging from accidental findings on routine radiography to life-threatening respiratory and cardiovascular compromise, which can also present as gigantic intrathoracic germ cell tumors, as in our case.

Case report: A 30-year-old male patient, not known to have any chronic illness, was referred from TB hospital center due to history of dyspnea, cough, and loss of appetite with weight loss for more than four months and no history of chest pain or hemoptysis. Chest X-ray was performed and showed complete obliteration of the right side of thorax; pleural effusion was suspected, and pleural TB and empyema were diagnosed. He was started on a tuberculosis drugs, antibiotics, and received chest drain with a slightly bloody fluid. Patient did not improve and was referred to our hospital. Computed hospital of chest with contrast revealed a very large mass obliterating the right side of chest, pushing the trachea and mediastinum to the left side with minimal effusion on both sides. Pleural US revealed mass and effusion but no empyema. Differential diagnosis was mediastina mass, adenocarcinoma, thymic carcinoma, lymphomas, fibroma, or fibrosarcoma. US guided transthoracic fine needle biopsy from the right-side mass revealed mixed germ cell tumor. The patient's condition had rapidly deteriorated prior to confirming the diagnosis or starting with treatments and died because of difficult airway breathing due to deviated and compressed airway and possible pneumothorax after transthoracic biopsy.

Conclusion: Germ cell tumors are aggressive and rapidly growing cancers. The literature reports the nature of the extragonadal mediastinal germ cell tumor as appearing as a giant mass occluding the whole lung, compressing the great vessels, and adhering to the chest wall, pericardium, and lung, as in our case. This leads to a worse prognosis. The estimated event-free survival at 10 years after combined treatment is $80.4 \%$. Chemotherapy, debulking, and pneumoctomy are the treatments for such cases.

\section{MGMT, BRCA1 and MEG3 methylation status in triple-negative breast cancer}

\section{Sylwia Paszek ${ }^{1}$, Agnieszka Kołacińska ${ }^{2,3}$, Marcin Braun $^{4}$, Ewa Kaznowska ${ }^{1}$, Dorota Jesionek- Kupnicka ${ }^{4}$, Edyta Barnaś ${ }^{1}$, Izabela Zawlik ${ }^{1}$}

\author{
${ }^{1}$ Faculty of Medicine, University of Rzeszow, Kopisto 2a, Rzeszow 35-959, Poland. \\ ${ }^{2}$ Department of Head and Neck Cancer Surgery, Medical University of Lodz, Paderewskiego 4, Lodz 93-509, \\ Poland. \\ ${ }^{3}$ Breast Unit, Cancer Center, Copernicus Memorial Hospital, Pabianicka 62, Lodz 93-513, Poland. \\ ${ }^{4}$ Department of Pathology, Chair of Oncology, Medical University of Lodz, Paderewskiego 4, Lodz 93-509, \\ Poland.
}

Background and aim: Breast cancer is one of the most common cancers in women worldwide. The most severe type of breast cancer is triple-negative breast cancer (TNBC) due to unfavorable clinical course 
and poor prognosis. The development of cancer is often associated with dysregulation of epigenetic mechanisms, including DNA methylation. The aim of our study was to evaluate MGMT (O6-methylguanine DNA methyltransferase), BRCA1 (breast cancer 1), and MEG3 (maternally expressed 3) methylation in TNBC.

Experimental procedure: In this study, 44 TNBC patients were included. The methylation status of the $M G M T, B R C A 1$, and MEG3 promoter regions were analyzed by methylation-specific PCR.

Results: MGMT, BRCA1, and MEG3 promoter methylation was found in $70.4 \%, 61.3 \%$, and $61.3 \%$ of TNBC patients, respectively. Moreover, we showed that the frequency of $M G M T$ and $B R C A 1$ methylation is higher in older patients compared to younger patients ( $P$-value for $M G M T$ is $P=0.0194$ and for $B R C A 1$ is $P=$ 0.0188). Additionally, in one of TNBC patient with glandular and squamous histopathological components, it was shown that the promoter status of all analyzed genes changed from methylated to unmethylated after chemotherapy of this patient.

Conclusion: The high frequency of $M G M T, B R C A 1$, and $M E G 3$ methylation indicates that epigenetic changes are important mechanisms in breast cancer. Moreover, our results indicate that $M G M T$ and $B R C A 1$ methylation may have greater impact in the development of breast cancer in older patients compared to younger patients.

\section{Sphingosine kinase 2 in oral squamous cell carcinoma}

\section{Lais Brigliadori Fugio, Andréia Machado Leopoldino}

Department of Clinical Analysis, Toxicology and Food Sciences, School of Pharmaceutical Sciences of Riberião Preto, University of São Paulo, Ribeirão Preto, SP 13010, Brazil.

Background and aim: Sphingosine kinase 2 (SK2) is one of the enzymes responsible for producing sphingosine-1-phosphate $\left(\mathrm{S}_{1} \mathrm{P}\right)^{[1]}$. Recently, $\mathrm{SK}_{2}$ has been associated with protective autophagy and survival, and regulation of $\mathrm{p} 21$ in breast and colon cancer cells ${ }^{[2,3]}$. However, the role of SK2 in oral squamous cell carcinoma (OSCC) is still unclear. Thus, our study aimed to investigate the involvement of SK2 in autophagy and proliferation in OSCC cells.

Experimental procedure: $\mathrm{HN}_{13}$ and $\mathrm{HN}_{12}$ (OSCC) cell lines were transduced with short hairpin RNA interference against SK2 and a lentiviral vector containing cDNA for SK2, respectively. Cell cycle analyses were performed by propidium iodide staining and flow cytometry. Western blotting and immunofluorescence assays were adopted to analyze protein levels and cellular distribution.

Results: HN13 cells with SK2 knockdown showed a decrease of pAkt, c-MYC, and LC3 levels (an autophagy marker) while p21 was increased. SK2 knockdown in HN13 cells caused cell arrest in S phase with reduction of the cells in G2/M. SK2 overexpression in HN12 cells led to an increase of pAkt, c-Myc, and LC3 levels.

Conclusion: Our work is the first to demonstrate the role of SK2 in proliferation and autophagy in OSCC cells. Other studies are in progress to understand the molecular mechanism underlying the role of SK2 and its potential as a target.

Financial support: FAPESP (grant: 2016/19103-2; scholarship: 2018/14225-8, CAPES, CNPq- Brazil). 


\title{
REFERENCES
}

1. Neubauer HA, Pitson SM. Roles, regulation and inhibitors of sphingosine kinase 2. FEBS J 2013;280:5317-36.

2. Sankala HM, Hait NC, Paugh SW, Shida D, Lépine S, et al. Involvement of sphingosine kinase 2 in p53-independent induction of p21 by the chemotherapeutic drug doxorubicin. Cancer Res 2007;67:10466-74.

3. Beljanski V, Knaak C, Smith CD. A novel sphingosine kinase inhibitor induces autophagy in tumor cells. J Pharmacol Exp Ther 2010;333:454-64.

\section{DNA methylation markers for noninvasive detection of early stage colorectal cancer}

\author{
Yanqun Liu' ${ }^{1}$ Min Hoe Chew ${ }^{1}$, Wah Siew Tan ${ }^{1}$, Choong Leong Tang ${ }^{1}$, Yi Zhao $^{2}$ \\ ${ }^{1}$ Department of Colorectal Surgery, Singapore General Hospital, Singapore 169608, Singapore. \\ ${ }^{2}$ Department of clinical Research, Singapore General Hospital, Singapore 169608, Singapore.
}

Background and aim: Colorectal cancer (CRC) is the most common cancer in Singapore. Earlier detection enhances chances of a cure and facilitates reducing CRC mortality rates. Colonoscopy is currently the gold standard for CRC diagnosis, but a somewhat troublesome and invasive procedure makes its acceptance not high in the general public as a screening tool. Epigenetic silencing of tumor-related genes by promoter methylation is common in CRC, but no biomarker has been proven to be individually of sufficient sensitivity or specificity in routine clinical practice. The aim of this study was to identify tumor-derived methylated genes in the serum of stage IIA CRC and assess their diagnostic potentials for early stage of colorectal cancer.

Experimental procedure: In this prospective study, DNA methylation levels were measured by quantitative methylation-specific PCR. Two genes ( $P P_{1} R_{3} C$ and $\left.A D H F E 1\right)$ were first investigated in serum samples of an exploratory set of stage IIA CRC case-controls. Methylation results were verified in the sera of a test set compromising 50 stage IIA cases and 50 age- and gender-matched healthy controls. The receiver operating characteristic curve was constructed for assessment of assay performance.

Results: Serum methylation levels of $P P P_{1} R 3 C$ and $A D H F E 1$ were significantly higher in stage IIA patients as compared to healthy controls (both $P<0.001$, Mann-Whitney $U$ test). Areas under the receiver operating curve (AUCs) using serum methylation levels of PPP1R3C and ADHFE1 were 0.60 [95\% confidence interval (CI), 0.48-0.71] and 0.73 (95\%CI, 0.62-0.83), respectively. At a specificity of $80 \%$, the assay sensitivities of methylated $P P P_{1} R 3 C$ and $A D H F E 1$ were $26 \%$ and $56 \%$, respectively.

Conclusion: Serum methylation levels of ADHFE1 might be useful for minimally invasive detection of early stage II colorectal cancer. Validation studies in larger and independent cohorts and identification of additional markers are necessary.

\section{Quantification of HER2 protein using multiple reaction monitoring-mass spectrometry in formalin-fixed paraffin-embedded (FFPE) breast cancer tissue specimens}

\author{
Misol Do ${ }^{1}$, Hyunsoo Kim², Han Suk Ryu ${ }^{3}$, Youngsoo Kim ${ }^{1,2}$ \\ ${ }^{1}$ Departments of Biomedical Sciences, Seoul National University College of Medicine, Seoul 03080, Korea. \\ ${ }^{2}$ Departments of Biomedical Engineering, Seoul National University College of Medicine, Seoul 03080, Korea. \\ ${ }^{3}$ Departments of Pathology, Seoul National University College of Medicine, Seoul 03080, Korea.
}

Human epidermal growth factor receptor 2 (HER2) protein is often overexpressed in breast cancer and is correlated with a worse prognosis; thus, accurate detection of HER2 by using optimum techniques is 
crucial to provide appropriate cares for patients. However, no technique is the universal gold standard to detect accurate HER2 status. In this context, we established a multiple reaction monitoring (MRM) assay to quantitate HER2 protein that improves upon existing methods in differentiating between each HER2 status in FFPE tissue specimens. We developed a targeted proteomic assay based on multiple reaction monitoring-mass spectrometry (MRM-MS) and quantified levels of HER2-MRM protein in breast cancer FFPE tissues.

We analyzed a total of 210 breast cancer FFPE tissue specimens, which were comprised of HER2 $0(n=30)$, HER2 $1+(n=30)$, HER2 2+FISH- $(n=61)$, HER2 2+FISH+ $(n=59)$, and HER2 $3+(n=30)$. We applied normalization factors that can represent the tumor size to simplify the overall experimental work-flow and raise the accuracy and precision of the results of HER2 quantification. In this context, the ratio between the quantification data of HER2 peptides by MRM assay and the normalization factor can be a new factor for determining HER2 status.

To select the most suitable normalization factor that can differentiate ambiguous IHC results of HER2 (HER2 2+FISH- vs. HER2 2+FISH+), which cannot be distinguished by IHC, area under the receiver operating curve (AUROC) values were calculated by using each normalized value of the 120 HER2 2+ samples. To determine whether the data generated by MRM matched with the data obtained by IHC and FISH scores, the quantitative data of a HER2 peptide normalized by a Junctional adhesion molecule A (JAM1) peptide with the highest AUROC values were used. The Mann-Whitney $U$ test determined that significant differences were found in all HER2 and FISH groups, and especially the MRM data can distinguish between HER2 2+FISH- and HER2 2+FISH+ $(P<0.000)$, which cannot be differentiated by IHC. In addition, the MRM data distinguished the HER2 positive group that was expected to benefit from trastuzumab therapy from the HER2 negative group $(P<0.000)$.

We developed an experimental work-flow that is simple and clear enough to automate by introducing normalization factors for accurate HER2 status determination through MRM assay. The MRM assay that we developed clearly distinguished the equivocal HER2 status that could not be classified by the conventional method, IHC, as well as the overall HER2 classification. Our developed assay using MRM for determining HER2 status would provide clinicians with valuable diagnostic information and ensure that all patients whose breast cancers express HER2 proteins have the opportunity to receive proper treatment.

\title{
41. Amino-functionalized nanoparticles promote toxicity in ovarian cancer cells by impinging on autophagy
}

\section{Alessandra Ferraresi ${ }^{1}$, Christian Seca ${ }^{1}$, Suratchanee Phadngam ${ }^{1,2}$, Chiara Vidoni $^{1}$, Marco Palminteri ${ }^{1}$, Ciro Isidoro ${ }^{1}$}

\author{
${ }^{1}$ Laboratory of Molecular Pathology and Nanobioimaging, Department of Health Sciences, Università del \\ Piemonte Orientale, Via Solaroli 17, Novara 28100, Italy. \\ ${ }^{2}$ Department of Radiologic Technology, Faculty of Associated Medical Sciences, Chiang Mai University, Chiang \\ Mai 50200, Thailand.
}

Background: In the last decades, nanotheranostics has obtained great attention for its potential application in the biomedical field by combining multimodal imaging along with selective targeting therapy on the same nanoplatforms ${ }^{[1]}$. However, the contributions of metabolic, genetic, or epigenetic features of tumor cells and tumor microenvironment in the cellular response to the nanoparticles have not been fully $\operatorname{addressed~}^{[2]}$. 
Aim: We investigated the cellular stress response to polystyrene nanoparticles (PS-NPs) functionalized with amino groups in two ovarian cancer cell models differing in the expression, among others, of relevant proteins involved in endocytosis (caveolin-1, CAV-1) and in pro-survival/pro-death pathways (PTEN and TP53).

Results: $\mathrm{NH}_{2}$-PS-NPs were toxic in both cell lines, leading to primary necrosis that was time- and dosedependent, yet with different mechanisms of toxicity. In OVCAR3 cells, which are PTEN and TP53 mutated and CAV-1 deficient, autophagy was insufficient to protect the cells from $\mathrm{NH}_{2}$-PS-NPs toxicity. Autophagy inducers prevented while autophagy gene silencing exacerbated $\mathrm{NH}_{2}$-PS-NPs-induced cell death. By contrast, in OAW42 cells, which express wild-type PTEN, TP53, and CAV-1, $\mathrm{NH}_{2}$-PS-NPs strongly impaired autophagosome formation and increased production of the mitochondrial anion superoxide, resulting in ATG4 inactivation. Accordingly, resveratrol, a nutraceutical known to inhibit the formation of anion superoxide, rescued ATG4-mediated autophagy and reduced $\mathrm{NH}_{2}$-PS-NPs toxicity.

Conclusion: Taken together, our findings point out the relevance of the genetic background of target cells, which determines the type and consequences of the stress response elicited by the NPs. Our data outline the necessity of a better assessment of the genetic/epigenetic and metabolic status of the target cells when designing theranostics for cancer therapy, in full agreement with the principle of personalized medicine.

\section{REFERENCES}

1. Prasad M, Lambe UP, Brar B, Shah I, J M, et al. Nanotherapeutics: an insight into healthcare and multi-dimensional applications in medical sector of the modern world. Biomed Pharmacother 2018;97:1521-37.

2. Nabil G, Bhise K, Sau S, Atef M, El-Banna HA, et al. Nano-engineered delivery systems for cancer imaging and therapy: Recent advances, future direction and patent evaluation. Drug Discov Today 2018;24:462-91.

\section{Glucose-dependent autophagy control of cancer cell migration}

\section{Chiara Vidoni ${ }^{1}$, Alessandra Ferraresi ${ }^{1}$, Letizia Vallino ${ }^{1}$, Eleonora Secomandi ${ }^{1}$, Andrea Esposito', Carlo Girone ${ }^{1}$, Danny N. Dhanasekaran ${ }^{2}$, Ciro Isidoro ${ }^{1}$}

\section{${ }^{1}$ Laboratory of Molecular Pathology and Nanobioimaging, Department of Health Sciences, Università del Piemonte Orientale "A. Avogadro", Via Solaroli 17, Novara 28100, Italy. \\ ${ }^{2}$ Stephenson Cancer Center and Department of Cell Biology, The University of Oklahoma Health Sciences Center, Oklahoma City, OK 73104, USA.}

Background and aim: Because of its aggressiveness and its diagnosis at very late stage, Ovarian cancer ${ }^{[1]}$ remains one of the main leading causes of death among women ${ }^{[2]}$. IL- 6 is an inflammatory cytokine over-expressed in serum and ascitic liquid of ovarian cancer-affected patients ${ }^{[3]}$. One of the hallmarks of cancer is the so-called Warburg effect, which consists of an alteration of glucose metabolism ${ }^{[4]}$. The goal was to investigate the mechanisms underlying the involvement of glycolysis and its mechanistic link with autophagy in cancer cell migration.

Experimental procedure: To mimic a pro-inflammatory tumor microenvironment, we treated ovarian cancer cells with IL-6, in absence or presence of glucose. To examine the molecular pathways linking glycolysis and autophagy in cell motility, we employed the metabolically inert glucose analog 2-Deoxy Glucose (2-DG). Additionally, we used resveratrol (RV), a nutraceutical with anti-cancer properties known to interfere with the utilization of glucose. 
Results: We found that glucose is necessary for cell migration, with IL-6 promoting glucose uptake and cell motility. On the contrary, inhibiting glucose uptake or its utilization blocks cancer cell migration while upregulating autophagy.

Conclusion: Our data indicate that the up-regulation of autophagy promoted by glucose deprivation hampers ovarian cancer cell migration.

\section{REFERENCES}

1. Hollis RL, Gourley C. Genetic and molecular changes in ovarian cancer. Cancer Biol Med 2016;13:236-47.

2. Siegel RL, Miller KD, Jemal A. Cancer statistics, 2017. CA Cancer J Clin 2017;67:7-30.

3. Plante M, Rubin SC, Wong GY, Federici MG, Finstad CL, et al. Interleukin-6 level in serum and ascites as a prognostic factor in patients with epithelial ovarian cancer. Cancer 1994;73:1882-8.

4. Soga T. Cancer metabolism: key players in metabolic reprogramming. Cancer Sci 2013;104:275-81.

\section{Trousseau's syndrome in association with lung adenocarcinoma}

\section{Abdulrahman Hakami}

Jazan University, Jazan 45142, KSA.

Background: Trousseau's syndrome (TS) is a hypercoagulability manifestation of paraneoplastic syndrome, known as a variant of cancer-associated thrombosis and defined as a migratory thrombophlebitis found typically in patients with an underlying malignancy. TS commonly occurs in pancreatic cancer $(24 \%)$, lung cancer $(20 \%)$, prostate cancer (13\%), and stomach cancer (12\%), followed by breast and colon cancer.

Case presentation: Here, we describe the case of a 50-year-old male patient, who is a nonsmoker. During a checkup for work, he was found to have a highly positive Mantoux test (TBT), thus a chest X-ray was ordered. He had a previous chronic history of burning sensation in both feet, responding to analgesic drugs. There was no history of shortness of breath,r cough, fever, night sweating, weight loss, loss of appetite, or fatigue. Auscultation of chest X-ray revealed a mass in the left upper lobe of lung. The computed tomography (CT) of chest showed a $5.5 \mathrm{~cm} \times 4.3 \mathrm{~cm}$ left lingual superior segment lobulated mass with left hilar and mediastinal lymph node enlargement. In addition, the CT reported bone metastasis in vertebra, confirming the bone scan. Tumor markers were negative. CT guided biopsy for this lesion in the left upper chest was performed and the histopathology result showed poorly differentiated adenocarcinoma. The following molecular studies were negative: epidermal growth factor receptor (EGFR), anaplastic lymphoma kinase, ROS, and PD-1. The patient was referred to the oncology center as a case of lung adenocarcinoma with distant metastasis, stage T4bN2bM1, and started in cisplatin and Alimta chemotherapy. Spiral CT revealed incidental finding of multiple filling defects, indicating segmental pulmonary embolism. Due to leg pain, Doppler of lower limb was also performed, showing deep venous thrombosis in the left limb. Enoxaparin was commenced at full dose. This case report indicated a TS cancer-associated thrombosis. The patient, after receiving the first cycle of chemotherapy, was discharged on enoxaparin, was stable, and returned to his job.

Conclusion: TS, a paraneoplastic manifestation, must be considered in patients with advanced stages of cancer, regardless of the primary site of the cancer. In lung cancer, paraneoplastic syndrome presents more frequently with small cell carcinoma (in 10\% of patients), but, regarding TS in the literature, previous cases reported adenocarcinoma was the most prevalent histology associated with thrombosis. 


\title{
44. Delphinidin chloride and its hydrolytic metabolite gallic acid promote differentiation of regulatory $T$ cells and have an anti-inflammatory effect on the allograft model
}

\author{
Ki Hyeob Hyun, Ki Cheol Gil, Kwang Woo Hwang
}

College of Pharmacy, Chung-Ang University, Seoul 06974, Korea.

Regulatory T cells (Tregs) control the reactivity of other $\mathrm{T}$ cells to prevent excessive inflammatory responses. They also play a role in preventing autoimmune diseases; however, when they are overproduced, they decrease vital immunity, which can lead to invasion of external pathogens. Therefore, it is most important in preventing the development of immune diseases to maintain the homeostasis of these cells. Delphinidin chloride is an anthocyanidin and known to have antioxidant activities. However, its structure is very unstable and easily decomposed. One of these degradation products is gallic acid, which also has antioxidant effects. In this study, we examined the effect of these materials on Tregs in controlling immune response. It was found that these materials further promote differentiation into Tregs, and TGF- $\beta$ and IL-2 related signals are involved in this process. Furthermore, it was verified that a variety of immunosuppressive proteins were secreted more, and the function of induced Tregs was also increased. Finally, in the allograft model, we could find a decrease in activated $\mathrm{T}$ cells when these materials were treated because they increased differentiation into Tregs. Therefore, these two materials are expected to become new candidates for the treatment of diseases caused by excessive activation of immune cells, such as autoimmune diseases.

Practical application: Delphinidin, a kind of anthocyanin rich in pigmented fruits, and its hydrolytic metabolite, gallic acid, are known to have antimicrobial and antioxidant properties. In this experiment, it was shown that delphinidin and gallic acid had an effect of increasing the differentiation of regulatory $\mathrm{T}$ cells, and the effect of suppressing the function of memory $\mathrm{T}$ cells was also observed. Due to these functions, delphinidin and gallic acid might have the potential to be used as immune suppressive agents in organ transplant and autoimmune disease patients or be a model for food development associated with the immune system.

\section{Association between heavy metal cadmium and the warburg effect in breast cancer - preliminary results}

\author{
Ewa Jabłońska ${ }^{1}$, Joanna Roszak², Beata Janasik ${ }^{3}$, Monika Lesicka ${ }^{1}, K^{2}$ Kornelia Kowalczyk ${ }^{2}$, Edyta \\ Wieczorek ${ }^{1}$, Leszek Kalinowski ${ }^{4}$, Jarosław Skokowski ${ }^{4}$, Wojciech Wąsowicz ${ }^{3}$, Edyta Reszka ${ }^{1}$ \\ ${ }^{1}$ Department of Molecular Genetics and Epigenetics, Nofer Institute of Occupational Medicine, Lodz 91-348, \\ Poland. \\ ${ }^{2}$ Department of Toxicology and Carcinogenesis, Nofer Institute of Occupational Medicine, Lodz 91-348, \\ Poland. \\ ${ }^{3}$ Department of Biological and Environmental Monitoring, Nofer Institute of Occupational Medicine, Lodz 91- \\ 348, Poland. \\ ${ }^{4}$ Central Bank of Tissues and Biological Material, Medical University of Gdansk, Gdansk 80-210 Poland.
}

Background and aim: Warburg effect is a cancer hallmark described as reprogramming of energy metabolism, in which cells produce energy mainly due to glycolysis instead of oxidative phosphorylation. The Warburg effect is extremely important for the survival of tumor cells, particularly under hypoxia, but it may also occur under aerobic conditions (hence, it is called aerobic glycolysis). Although Warburg effect 
was discovered almost 100 years ago, it is still not known whether it is a cause or a consequence of cancer. Interestingly, there are few studies investigating the association between the known carcinogenic factors and the Warburg effect. The aim of this study was to analyze the association between carcinogenic metal cadmium (Cd) and the Warburg effect in breast cancer.

Experimental procedure: We conducted an observational study on 100 women with breast cancer, from whom fragments of tumor tissue and tumor-adjacent tissue were collected, in order to compare Cd contents and molecular effect of the Warburg effect. In both types of tissue, we determined Cd content and the expression of mRNA of HIF-1 $\alpha$ (the key driver of the Warburg effect) and other proteins associated with the Warburg effect (including glucose transporters, glycolytic enzymes, or kinases regulating glycolysis). In addition, urinary $\mathrm{Cd}$ concentration as a marker of environmental exposure was analyzed. To investigate the effect of $\mathrm{Cd}$ on the Warburg effect in vitro, we analyzed molecular and metabolic markers of the Warburg effect (lactate concentration and pyruvate kinase activity) in MCF-7 cells exposed to nontoxic, environmentally relevant concentrations of $\mathrm{Cd}$ for $72 \mathrm{~h}$ (short term exposure) and six months (imitation of chronic exposure to $\mathrm{Cd}$ ).

Results: In the preliminary study of 15 patients, we observed significant positive correlation between urinary Cd concentration and the expression of HIF- $1 \alpha$, in both tumor $(r=0.80, P<0.001)$ and tumoradjacent tissues $(r=0.75, P<0.001)$. Cd content in tumor tissue was also significantly correlated with the expression of PDK1 (pyruvate dehydrogenase kinase $1 ; r=0.48, P<0.001$ ). Preliminary data analysis of MCF7 cell line showed that HIF- $1 \alpha$ expression was also significantly increased upon Cd exposure (1-20 $\mu \mathrm{M})$.

Conclusion: Preliminary results of this study may suggest a possible link between Cd exposure and the molecular effects of the Warburg effect. Complete data analysis, including metabolic markers, will allow formulating final conclusions.

\section{The oyster can adapt to a harsh environment in the marine coast: does it mimic cancer cells?}

\section{Charlotte Corporeau ${ }^{1}$, Catherine Brenner ${ }^{2}$, Nathalie Mazure ${ }^{3}$}

${ }^{1}$ Team supported by the ARC foundation, Ifremer, Laboratoire des sciences de l'Environnement Marin (UMR 6539, LEMAR), Plouzané 29280, France.

${ }^{2}$ INSERM U1180, Université Paris Sud, 5 rue J.B. Clément, Châtenay-Malabry 92296, France.

${ }^{3}$ INSERM U1065, Centre Méditerranéen de Médecine Moléculaire, 151 route St Antoine de Ginestière, Nice 06204 , France.

Background and aim: The tumor physical microenvironment is extreme. Interestingly, the rocky intertidal zone is among the most physically harsh environments on Earth. The oyster Crassostrea gigas, living in this habitat, is among the champions of physiological adaptation to extreme environments. Our hypothesis is that environmental adaptation of oyster cells can mimic cancer cells inside the tumor.

Experimental procedure: Oysters were challenged to three extreme environments in the field, at high, medium, and low bathymetric levels. Biochemical analysess were done to identify the environmental responses.

Results: At two times during the experiment, we sampled all the organs of oysters in the field, extracted total proteins from flesh, and performed laboratory analysis, in order to obtain a rapid picture of metabolic 
activities linked with extreme environmental responses. The first results demonstrated that challenged oysters in high/medium bathymetry exhibited a low weight gain, increased hexokinase activity, and increased mitochondrial functioning. We also quantified an up-regulation of AMP-activated protein kinase (AMPK) activation, a key energy sensor that controls glucose, lipid, and protein metabolism in C. gigas. Interestingly, up-regulation of AMPK was initially reported as a hallmark of cancer cells to support the high-energy demand of highly proliferative cells.

Conclusion: We propose the oyster as a new model for cancer research, to identify mechanisms underlying the ability of cells to adapt to a harsh environment. The oyster is a marine invertebrate that evolved 500 million years ago and we are convinced that it could help us to identify common ancestral pathways for cell adaptation to a harsh environment, for a better understanding of cancer cells functioning inside the tumor.

\section{Resveratrol-induced modulation of non-coding RNA in ovarian cancer cells}

\section{Letizia Vallino ${ }^{1}$, Alessandra Ferraresi ${ }^{1}$, Chiara Vidoni $^{1}$, Claudia Lora ${ }^{1}$, Chinmay Maheshwari $^{1}$, Danny N. Dhanasekaran ${ }^{2}$, Ciro Isidoro'}

${ }^{1}$ Laboratory of Molecular Pathology, Department of Health Sciences, Università del Piemonte Orientale "A. Avogadro", Via Solaroli 17, Novara 28100, Italy.

${ }^{2}$ Stephenson Cancer Research Center, University of Oklahoma City, Oklahoma City, OK 73104, USA.

Background and aim: Ninety percent of the human genome is transcribed, of which only $2 \%$ encodes proteins; the remaining sequences enclose non-coding genes ${ }^{[1,2]}$. Non-coding RNAs (ncRNAs) play a crucial role in the regulation of several biological processes and their dysregulation may influence cancer development, functioning as oncogenes or tumor suppressors. ncRNAs generally are divided into two main groups based on size: long non-coding RNAs (lncRNAs) of about 200 nucleotides, acting as positive or negative transcription modulators, and microRNAs (miRNAs) of about 20-22 nucleotides, acting as posttranscriptional silencing molecules ${ }^{[3,4]}$. The aim of our work was to investigate whether Resveratrol (RV), a polyphenolic compound with anticancer properties ${ }^{[5]}$, could modulate ncRNAs in ovarian cancer cell lines.

Experimental procedure: Ovarian cancer cells were treated with RV $(100 \mu \mathrm{M})$. Total RNA was isolated from the cells and mRNA was amplified and labeled. Labeled specimens were fragmented and hybridized to Human Whole Genome Oligo Microarrays. One hundred nanograms of total RNA were treated following the miRNA microarray protocol. RNA was dephosphorylated, denaturated, ligated, and labeled. Samples were hybridized to Human miRNA Microarray. DIANA TOOLS was used to retrieve predicted microRNA targets and Gene Ontology for predicting their involvement in biological processes.

Results: We show that RV modulates non-coding transcripts that impact on cancer cell features.

Conclusion: Our data support the view that RV treatment can be effective in cancer therapy on regulating epigenetic mechanisms involved in cancer development.

\section{REFERENCES}

1. Stein LD. Human genome: end of the beginning. Nature 2004;431:915-6.

2. Ponting CP, Belgard TG. Transcribed dark matter: meaning or myth? Hum Mol Genet 2010;19:R162-8.

3. Mendell JT. MicroRNAs: critical regulators of development, cellular physiology and malignancy. Cell Cycle 2005;4:1179-84.

4. Wapinski O, Chang HY. Long noncoding RNAs and human disease. Trends Cell Biol 2011;21:354-61.

5. Elshaer M, Chen Y, Wang XJ, Tang X. Resveratrol: An overview of its anti-cancer mechanisms. Life Sci 2018;207:340-9. 


\section{The microbiota-derived metabolite butyrate inhibits colorectal cancer cell migration via modulation of autophagy}

Eleonora Secomandi, Chiara Vidoni, Alessandra Ferraresi, Giulia Camurani, Ciro Isidoro

Laboratory of Molecular Pathology and Nanobioimaging, Department of Health Sciences, Università del Piemonte Orientale, Via Solaroli 17, Novara 28100, Italy.

Background: Colorectal cancer (CRC) is the third most common cause of cancer deaths worldwide. The etiology of CRC involves host genetic predisposition and environmental factors, among which the diet plays an important role. The proportion of dietary fiber and meat consumed influences the composition of intestinal microbiota, which get energy from ingested food ${ }^{[1]}$. One of the main metabolites produced by gut microbiota is the short chain fatty acid butyrate. Butyrate exerts a beneficial role in the maintenance of intestinal epithelium integrity through various mechanisms ${ }^{[1]}$. Autophagy is the main cellular process that promotes a balanced macromolecular turnover and guarantees cell homeostasis ${ }^{[2]}$.

Aim: We studied the anti-migratory and anti-inflammatory properties of butyrate, a probiotic metabolite, in a colorectal cancer cellular model. Furthermore, we investigated the molecular pathways underlying these effects, with a particular focus on autophagy.

Experimental procedure: HCT116 colorectal cancer cells were treated with 5-mM sodium butyrate and 50-ng/mL interleukin-6 (IL-6). To study cell motility, a wound healing scratch assay was performed. Cellular homogenates were employed for protein expression studies through Western blot analysis. Immunofluorescence was performed on fixed cells.

Results: We found that butyrate counteracts colorectal cancer cell migration, even in the presence of IL-6, a well known pro-inflammatory cytokine. This effect is accompanied with a reduced expression of activated STAT3 and Twist1. Furthermore, the probiotic metabolite prevents IL-6-induced expression of N-cadherin, a typical hallmark of epithelial-to-mesenchymal transition. In addition, butyrate strongly accelerates the autophagy flux, alone and in co-presence with IL-6, suggesting autophagy as a putative mechanism responsible for slowing down cell motility.

Conclusion: Taken together, our findings identified anti-cancer properties of butyrate, in particular its ability to counteract IL-6-induced colon cancer cell migration, by upregulating autophagy.

\section{REFERENCES}

1. Wu X, Wu Y, He L, Wu L, Wang X, et al. Effects of the intestinal microbial metabolite butyrate on the development of colorectal cancer. J Cancer 2018;9:2510-7.

2. Mizushima N, Levine B. Autophagy in mammalian development and differentiation. Nat Cell Biol 2010;12:823-30.

\section{Parasites and cancer}

\section{Omar M. Amin}

Parasitology Center Inc, Scottsdale, AZ 85259, USA.

This PowerPoint presentation is based on our work at Parasitology Center, Inc. (PCI), in Scottsdale, Arizona, USA and covers the diagnosis, pathology, relationships with cancer, and treatment of human 
parasitic infections in the United States based on our own patient history and testing. The conceptual thesis and practical observations of extensive damage of parasites to human tissues and the initiation of host defense strategies causing out of control cell divisions leading to metastasis is emphasized. A brief introduction to laboratory procedures, misdiagnoses/mistreatment, and impact on public health, especially cancer, is made. A systematic treatment of protozoan and helminth (worm) parasites follows, emphasizing epidemiology and exposure, symptoms, and gross pathology. Herbal and allopathic remedies including our own anti-parasitic herbal product Freedom/Cleanse/Restore are presented. All topics are illustrated with labeled pictures of the various kinds of parasites and their gross pathology in human tissues, when applicable. The presentation is followed by a brief discussion of case histories and treatment of intestinal pathogenic bacteria that usually cause GI symptoms similar to those caused by intestinal parasites.

\section{Role of biobanks in cancer research}

\section{Judita Kinkorova}

University Hospital in Pilsen and Charles University, Faculty of Medicine in Pilsen, Pilsen 304 60, Czech Republic.

Background and aim: Biobanks are an important tool for biomedical research and a pillar of personalized medicine. Biobanks are collections of biological material and the associated data and information stored in an organized system, for a population or a large subset of population (OECD definition) ${ }^{[1]}$. During last three decades, the role of biobanks has increased dramatically. They act as sources for a wide range of biomedical research and support the basic principles of personalized medicine. They contribute to prevention, early diagnosis, prognosis, right treatment, therapy monitoring, and optimal approach to a patient ${ }^{[2]}$. Cancer was and remains as one of the main causes of mortality and morbidity worldwide. The number of cancers, especially rare cancers, make cancer diseases serious candidates for personalized medicine approaches.

How can biobanks contribute to the process of cancer treatment?

Biobanks as sources of various human biological material from different patients, from different regions, males and females, young and elderly, with other comorbidities, before and after surgery, different social and environmental associations, different ethnic groups, etc., offer a wide range of samples for any type of research. Based on these characteristics, biobanks are a cornerstone for the discovery of new biomarkers, drugs, techniques, applications, and innovations ${ }^{[3]}$. On the other hand, data and information connected with samples are another source for modeling, data applications, and artificial intelligence applications, with respect to ethical, legal, and social issues. Personalized medicine principles guarantee the best possible approach to every patient, which means prevention, early diagnosis, treatment, and treatment monitoring. Biobanking is a phenomenon that is intrinsically based on international collaboration, including samples, data, and information exchange, as no one institution can cover all the challenges all over the world by offering biobanking. Biobanking requires a multidisciplinary international strategy.

Conclusion: Biobanks are efficient tools for new biomedical research and a personalized medicine approach to every patient and contribute to support healthcare systems, international collaboration, biomedical research, and innovation.

\section{REFERENCES}

1. Kinkorová J. Biobanks in the era of personalized medicine: objectives, challenges, and innovation: Overview. EPMA J $2016 ; 7: 4$.

2. Kinkorová J. The 39th annual conference: Immunoanalytical Days - Pilsen, Czech Republic, 11-13 March 2018. Biomark Med 2019;13:601-4.

3. Kinkorová J, Topolčan O. Biobanks in Horizon 2020: sustainability and attractive perspectives. EPMA J 2018;9:345-53. 


\section{Cancer chemoprevention with mitochondria-targeted compounds}

\section{Ming You}

Center for Disease Prevention Research and Department of Pharmacology and Toxicology, Medical College of Wisconsin, 8701 Watertown Plank Road, Milwaukee, WI 53226, USA.

We synthesized two mitochondria-targeted compounds, namely mito-honokiol (Mito-HNK) and mitolonidamine (Mito-LND), that facilitate their mitochondrial accumulation. This dramatically increases their potency and efficacy against highly metastatic lung cancer lines in vitro, orthotopic lung tumor xenografts, and brain metastases in vivo. Both Mito-HNK and Mito-LND are $>100$-fold more potent than their parent compounds in inhibiting cell proliferation and mitochondrial complexes, stimulating reactive oxygen species generation, and oxidizing mitochondrial peroxiredoxin-3. Interestingly, Mito-HNK appears to induce apoptosis via suppressing the phosphorylation of mitoSTAT3, while Mito-LND induces autophagic cell death via inactivating AKT/mTOR/p70S6K signaling. Both Mito-HNK and Mito-LND cause no toxicity in mice, even when administered for eight weeks at $>20$ times the effective cancer inhibitory dose. A highly synergistic effect is observed when combining the two compounds and its mechanistic basis is being vigorously pursued. Collectively, these findings show that mitochondrial targeting compounds are a promising preventive/therapeutic approach to mitigate lung cancer development and brain metastasis.

\section{The efficacy of ketogenic diet with concomitant intranasal perillyl alcohol as a novel strategy for therapy of recurrent glioblastoma}

\section{Juliana G. Santos1, Wanise S. Cruz1, Axel H. Schönthal2, Thereza Q. Santos3, Clovis O. Da Fonseca4}

${ }^{1}$ Department of Nutrition, Fluminense Federal University, Niteroi Rio de Janeiro 24220-900, Brazil.

${ }^{2}$ Keck School of Medicine, University of Southern California, Los Angeles, CA 90007, USA.

${ }^{3}$ Department of Cellular and Molecular Biology, Institute of Biology, Fluminense Federal University, Niteroi Rio de Janeiro 24220-900, Brazil.

${ }^{4}$ Service of Neurosurgery, Fluminense Federal University, Niteroi Rio de Janeiro 24220-900, Brazil.

Background: It has been hypothesized that persistent ketotic hypoglycemia might represent a potential therapeutic strategy against high-grade gliomas. Perillyl alcohol (POH) is a non-toxic, naturally-occurring, hydroxylated monoterpene that exhibits cytotoxicity against temozolomide-resistant glioma cells, regardless of O6-methylguanine-methyltransferase promoter methylation status. This study aimed to evaluate the toxicity and therapeutic efficacy of intranasal $\mathrm{POH}$ administered in combination with a ketogenic diet (KD) program for the treatment of patients with recurrent glioblastoma.

Patients and methods: Thirty-two patients were divided into two groups - KD or standard diet - both associated with intranasal $\mathrm{POH}$ ( $n=17$ and $n=15$, respectively). The nutritional status and anthropometric parameters of patients were measured. Patients who adhered to the KD maintained a strict dietary regimen, while receiving inhalation of $\mathrm{POH}$ (55 mg, four times daily) in an uninterrupted administration schedule for three months. Neurological examination and imaging analysis (magnetic resonance imaging) were used to monitor disease progression. Clinical toxicity and overall survival were correlated with tumor size, topography, extent of peritumoral edema, and frequency of seizures. In the KD patient, strict compliance with the KD was confirmed by measuring the levels of ketone bodies in the urine ( $9 / 17$ patients) three times per week. 
Results: After three months of well tolerated treatment, we observed a partial response in $77.8 \%$ (7/9 patients), stable disease in $11.1 \%(1 / 9)$, and $11.1 \%(1 / 9)$ presented with progressive disease. Among the patients assigned to the standard diet (control group), the partial response was 25\% (2/8 patients), stable disease was $25 \%(2 / 8)$, and progressive disease was $50 \%$ (4/8 patients). The patients assigned to the KD group presented with: reduced frequency of seizures, a slight increase in lean muscle mass, reduced serum lipid levels, and decreased low-density lipoprotein cholesterol (LDL-C) levels.

Conclusion: These results are encouraging and suggest that $\mathrm{KD}$ associated with intranasal $\mathrm{POH}$ may represent a viable option as an adjunct therapy for recurrent GBM. 